\title{
Sound scattering by a compact circular pore ${ }^{\hbar}$
}

\author{
C.Y. Kuo ${ }^{\mathrm{a}, *}$, R.L. Chern ${ }^{\mathrm{b}}$, C.C. Chang ${ }^{\mathrm{a}, \mathrm{b}}$ \\ ${ }^{a}$ Division of Mechanics, Research Center for Applied Sciences, Academia Sinica, 128, Nankang 115, Taipei, Taiwan, ROC \\ ${ }^{\mathrm{b}}$ Institute of Applied Mechanics, National Taiwan University, 1, Sec. 4, Roosevelt Road, Taipei 106, Taiwan, ROC
}

Received 20 September 2007; received in revised form 5 June 2008; accepted 8 June 2008

Handling Editor: L.G. Tham

Available online 3 August 2008

\begin{abstract}
The aim of this paper is to study the three-dimensional scattering of an oblique wave incident on a flanged circular compact pore of finite depth. The multipole structure with the scattering is resolved by the method of matched asymptotic expansion, where we assume smallness of $\varepsilon=k^{*} a^{*}$, the product of the incident wavenumber $k^{*}$ and the pore radius $a^{*}$. Two distinguished cases are solved: the rigid boundary condition and the pressure-release boundary condition. The study presents by far the most complete solutions to these problems, with the outer solution up to $O\left(\varepsilon^{5}\right)$ and the inner solution up to $O\left(\varepsilon^{2}\right)$. In particular, the sophisticated interplay between the pore depth and the incident angle is revealed in the different orders of solution. It is shown that the leading order of the outer wave field for both cases is $O\left(\varepsilon^{3}\right)$. For the rigid boundaries, there is one dipole dependent on the incident angle and one monopole. Interestingly, the monopole arises from the second-order interaction of the pore volume and the small but non-negligible compressibility in the inner field. This is one of the few examples analytically solvable to demonstrate this property. On the other hand, only one dipole is found for the pressure-release boundary. The next order in the outer solutions for both types of the boundary conditions is of $O\left(\varepsilon^{5}\right)$ and is shown to contain quadrupoles and octupoles. The multipole structures for both types of boundaries are tabulated, explicitly with the effects of the incident angle and the pore depth.
\end{abstract}

(C) 2008 Elsevier Ltd. All rights reserved.

\section{Introduction}

We investigate the scattering of acoustic waves incident on a small circular pore with a finite depth in a semi-infinite flat surface. The radius and the depth of the pore are both assumed to be much smaller than the wavelength of the incident waves. Under these circumstances, a multipole expansion is appropriate for understanding the mechanisms because of its ability to illustrate the physics in different orders of magnitude and to express the multipole moments, or strengths, explicitly in closed forms. The compactness of the pore is measured by a small parameter $\varepsilon$, which is defined by the product of the incident wavenumber $k^{*}$ and the radius of the pore $a^{*}$. The pore depth $\ell^{*}$, being compact, is, therefore, comparable to $a^{*}$.

\footnotetext{
This work is supported in part by National Science Council, Taiwan, Republic of China, under Grant 96-2221-E-001-001.

* Corresponding author.

E-mail address: cykuo06@gate.sinica.edu.tw (C.Y. Kuo).
} 
In the literature, only a few references are related to the present study. A very brief note was presented by Sato and Shirai [1] on sound wave transmission through ducts in thick walls. The diffraction of a twodimensional flanged duct with external incident waves has been solved numerically by Shenderov [2]. He formulated the scattering problem into an integral equation that is reduced to an infinite system of linear algebraic equations. Directivities and spectra of the scattering waves and interactions with the pipe resonant modes are addressed for a wide range of sound frequencies. Scharstein and Davis [3] studied the electromagnetic wave scattering of a two-dimensional subwavelength semi-circular trough in a ground plane. They also used the method of matched asymptotic expansion to solve the multipole structure of the scattering wave. Although the multipole expansion can be derived from these studies, the two-dimensional wave is different from the three-dimensional counterpart in that the colatitude variation does not exist in the former configuration. This variation introduces asymmetry of the multipole structure and generates new multipoles when the incident wave sheds obliquely on the pore and, to the authors' knowledge, has not yet been addressed.

A further motivation for the current study was that we noticed that recently, there has been renewed interest in electromagnetic waves incident on flat surfaces with periodic structures of small poles. For example, GarciaVidal et al. [4] have investigated the finite-difference time-domain (FDTD) calculation of the transmission of the wave through a single rectangular hole in a perfect conductor plate, and Garcia de Abajo and Saenz [5] have calculated the effective permittivity of a flat perfect conductor with such a pore structure to model surface plasmon on metal surfaces. Further development of the present theory along this direction would provide an alternative point of view on both the near field and the far field radiation mechanisms of these emerging interests.

The significant difference between the present configuration and sound emission from a duct is that the fluid mass near the pore exit resists greatly the penetration of the waves being propagated into the duct. The leading order monopole, $O(1)$, for sound emitted from the duct due to the mass flux crossing the pore exit, see Pierce [6, Chap. 7], is degenerated into higher orders for the present situation. Hence, multipoles at higher orders must be solved altogether. By using the method of matched asymptotic expansion, we can divide the flow into two regions: the inner flow and outer wave regions. The method similar to Crighton [7] and Kuo and Dowling [8] can be applied to obtain solutions up to $O\left(\varepsilon^{2}\right)$ and $O\left(\varepsilon^{5}\right)$ for the inner and outer regions, respectively.

We first demonstrate the solution for the rigid surface condition. For the inner solution, the leading order of the scattering field is a dipole of $O(\varepsilon)$ due to the incident angle. A monopole also exists in the solution but is shown to appear at $O\left(\varepsilon^{2}\right)$ due to the small but non-negligible fluid compressibility. However, the monopole and the dipole fields become comparable in the outer wave region and are combined into the leading order solution in this region, which is of $O\left(\varepsilon^{3}\right)$. This is one of the few examples analytically solvable to demonstrate this peculiar property of acoustic waves.

The same analytical method is also applicable to the same problem but with pressure-release surfaces. This boundary condition occurs for acoustics when sound is shed on free-moving surfaces, such as liquid surfaces. Though it is unlikely that any solid materials would reproduce this wave field acoustically, the derivation of the solution for this condition is still worthwhile because it is extendible to cope with other wave systems, such as electro-magnetic waves. The leading term in both inner and outer regions is a dipole perpendicular to the flanged flat surface.

In what follows, we describe the geometry of the problem in Section 2 and solve for the rigid boundary condition in Section 3. The details are provided as thoroughly as possible. In Sections 3.1 and 3.2, the firstand second-order inner pressure fields are solved, and in Sections 3.3 and 3.4, the matching process to the outer wave region is performed. In a parallel manner, the derivation for the pressure-release boundaries is presented in Section 4. Numerical calculation results for both types of boundaries and the prospects of future studies are provided in Sections 3.5, 4.3 and 5. The main results, the multipole structures, are tabulated in Tables 1 and 2, explicitly with the effects of the incident angle and the pore depth.

\section{Geometry and governing equations}

The problem of interest and its coordinate definition are sketched in Fig. 1. There is a circular pore with a finite depth drilled in a semi-infinite rigid domain. The pore has a radius $a^{*}$ and a finite depth $\ell^{*}$. An incident 


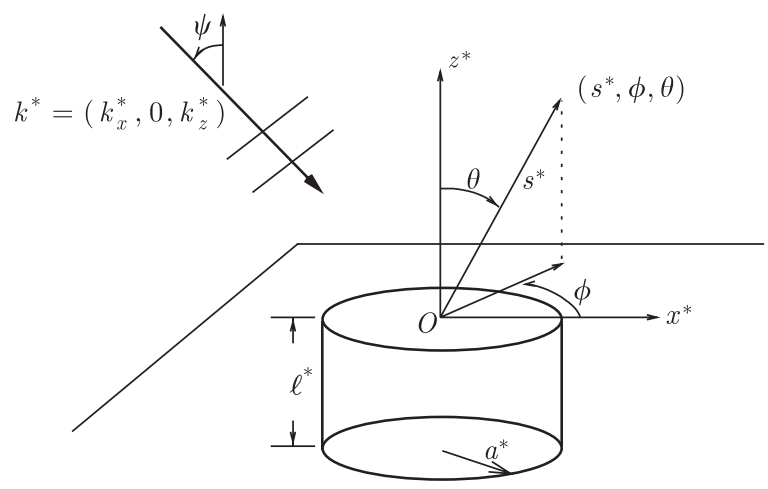

Fig. 1. The coordinate system and the incident wave.

plan wave is directed to the pore with an incident angle $\psi$. The coordinate is chosen such that $k_{y}^{*}=0$, i.e. symmetric with respect to the $x z$ plane. The spherical coordinate $\left(s^{*}, \phi, \theta\right)$ and the cylindrical coordinate $\left(\rho^{*}, \phi, z^{*}\right)$, with $\rho^{* 2}=x^{* 2}+y^{* 2}$ and $s^{* 2}=x^{* 2}+y^{* 2}+z^{* 2}$, are used on appropriate occasions.

The wave equation with a time harmonic proportional to $\exp \left(-\mathrm{i} \omega^{*} t^{*}\right)$ is

$$
\left(\nabla^{* 2}+k^{* 2}\right) p^{*}\left(\mathbf{x}^{*}, \omega^{*}\right)=0,
$$

where $p^{*}$ is the total sound pressure. Variables with an asterisk superscript ${ }^{*}$ denote the dimensional physical quantities. $\nabla^{* 2}$ is the Laplace operator and $k^{* 2}$ is $\omega^{* 2} / c^{* 2}$, where $\omega^{*}$ and $c^{*}$ are the sound frequency and the speed, respectively. The incident wave is described by $p_{\text {inc }}^{*}=I^{*} \exp \left(\mathrm{i} k_{x}^{*} x^{*}-\mathrm{i} k_{z}^{*} z^{*}\right)$, where $I^{*}$ is the pressure amplitude and $k_{x}^{*}=k^{*} k_{x}=k^{*} \sin (\psi)$ and $k_{z}^{*}=k^{*} k_{z}=k^{*} \cos (\psi)$.

There are two characteristic length scales: the wave length $1 / k^{*}$ for the outer region, far away from the pore, and the pore radius $a^{*}$ for the inner region, which contains the flow field in the pore and around the pore exit region. We normalize Eq. (1) with respect to the characteristic scales for both inner and outer regions:

$$
\left(\nabla_{i}^{2}+\varepsilon^{2}\right) p(\mathbf{x})=0 \quad \text { and } \quad\left(\nabla_{o}^{2}+1\right) P(\mathbf{X})=0,
$$

where $\mathbf{x}=\mathbf{x}^{*} / a^{*}, \mathbf{X}=k^{*} \mathbf{x}^{*}$. The sound pressure is normalized against $2 I^{*}$. Because the acoustic wave length is much larger than the pore radius, we have a small parameter $\varepsilon=k^{*} a^{*}$ such that $\mathbf{X}=\varepsilon \mathbf{X}$.

Interactions between the incident wave and the pore take place in the inner region. We consider two perfect conditions: the hard surface, or no penetration condition, i.e. $\partial p^{*} / \partial n^{*}=0$, and the pressure-release condition, $p^{*}=0$. The resultant inner flow, which acts as multipole acoustic sources with determinable strengths, is then matched to the outer wave field. The wave field that sees the singularities at the origin, cf. Eq. (35), is simply wave propagation over a flat surface with its boundary condition at the $Z=0$ plane. The matching process reveals the types of the multipole sources.

\section{Rigid reflective surface}

After normalizing the sound pressure by $2 I^{*}$, the external wave field, the incident wave and its reflection by the rigid plane is $p_{\text {ext }}=\cos \left(k_{z} Z\right) \exp \left(\mathrm{i} k_{x} X\right)$. It satisfies $\partial p / \partial z=0$ at $z=0$. This leads to the approximation in the inner flow region

$$
p_{\mathrm{ext}}=\left(1-\frac{\varepsilon^{2}}{2} k_{z}^{2} z^{2}\right)\left(1+\mathrm{i} \varepsilon k_{x} x-\frac{\varepsilon^{2}}{2} k_{x}^{2} x^{2}\right)+O\left(\varepsilon^{3}\right) .
$$

From Eqs. (2) $)_{1}$ and (3), it is suggested that the inner expansion for the total pressure is in the form of

$$
p(\mathbf{x})=1+\varepsilon p^{(1)}(\mathbf{x})+\varepsilon^{2} p^{(2)}(\mathbf{x})+O\left(\varepsilon^{3}\right) .
$$

The first term on the right-hand side is the leading term of the external field. It is a uniform field in the inner region. The rest of the terms contain the induced scattering fields and higher orders of the external field. 
Substitution of Eq. (4) into Eq. (2) 1 leads to the governing equations of the two orders

$$
\nabla_{i}^{2} p^{(1)}=0, \quad \nabla_{i}^{2} p^{(2)}+1=0, \quad \text { etc. }
$$

The equations also hold for the pressure in the pore region.

The inner region consists of two zones. They are one semi-infinite flow region, $z \geqslant 0$, and the cylindrical pore region, $\rho=1$, and $-\ell \leqslant z \leqslant 0$. Let us define the total pressure in the semi-infinite zone and the pore region as $p(\mathbf{x})$ and $p_{\text {pore }}(\rho, \phi, z)$, respectively. The inner solution can be constructed by matching the solutions of the two zones at the pore exit, $z=0$ and $\rho \leqslant 1$. This is typically known as a mixed boundary value problem. Mathematical theories and other applications of the relevant problems can be found in monographs, such as Sneddon [9] and Fabrikant [10].

\subsection{First-order inner solution}

Taking the first order as an example, we can express the total pressure for the semi-infinite zone $z \geqslant 0$

$$
\begin{aligned}
p^{(1)}(\rho, \phi, z) & =\mathrm{i} k_{x} x-\left.\frac{1}{2 \pi} \int_{0}^{2 \pi} \int_{0}^{1} \frac{\partial p^{(1)}\left(\rho_{0}, \phi_{0}, z_{0}\right)}{\partial z_{0}}\right|^{<} \frac{\rho_{0} \mathrm{~d} \rho_{0} \mathrm{~d} \phi_{0}}{\mathrm{~s}} \\
& =\mathrm{i} k_{x} x+p_{\mathrm{sc}}^{(1)}(\rho, \phi, z),
\end{aligned}
$$

where the term $\mathrm{i} k_{x} x$ is the spatial variation due to the external oblique incident wave. The second term, denoted as $p_{\mathrm{sc}}^{(1)}(\rho, \phi, z)$, is the near-field scattering, which is obtained by using the semi-infinite Green function with a vanishing normal derivative at $z=0$. We use the abbreviation $\mathrm{s}^{2}=\rho_{0}^{2}+\rho^{2}+z^{2}-2 \rho \rho_{0} \cos \left(\phi-\phi_{0}\right)$ for the square of the distance between two points $(\rho, \phi, z)$ and $\left(\rho_{0}, \phi_{0}, 0\right)$ in the cylindrical coordinate. The evaluation symbol $\left.\right|^{<}$indicates that the operand is evaluated in the region $\rho \leqslant 1$ at $z=0$ or $\rho_{0} \leqslant 1$ at $z_{0}=0$, whichever is appropriate.

Because the surface source, $\left.\left(\partial p^{(1)} / \partial z_{0}\right)\right|^{<}$, is still an unknown, it needs to be solved by matching with the pressure condition in the pore. For this purpose, we bring the observation point $(\rho, \phi, z)^{-}$to the pore exit, i.e. $\rho \leqslant 1, z=0$. At the exit, we have the pressure continuity, $\left.p_{\text {pore }}^{(1)}\right|^{<}=p^{(1)}=\mathrm{i} k_{x} x+\left.p_{\mathrm{sc}}^{(1)}\right|^{<}$, and the pressure derivative continuity, $\left.\left(\partial p_{\text {pore }}^{(1)} / \partial z\right)\right|^{<}=\left.\left(\partial p^{(1)} / \partial z\right)\right|^{<}$. With these conditions, we reach an integral equation

$$
\left.p_{\text {pore }}^{(1)}\right|^{<}=\mathrm{i} k_{x} x-\left.\frac{1}{2 \pi} \int_{0}^{2 \pi} \int_{0}^{1} \frac{\partial p_{\text {pore }}^{(1)}}{\partial z}\right|^{<} \frac{\rho_{0} \mathrm{~d} \rho_{0} \mathrm{~d} \phi_{0}}{\mathrm{~s}_{e}},
$$

which uses the pore pressure as unknowns. This is a Fredholm equation of the first kind. We have omitted the arguments $(\rho, \phi, z)$ in $p_{\text {pore }}^{(1)}$ and $\partial p_{\text {pore }}^{(1)} / \partial z_{0}$ and abbreviated $\mathrm{s}_{e}=\left.\mathbf{s}\right|_{z=0}$ for simplicity.

The integral equation, Eq. (6), can be solved elegantly by the method proposed by Fabrikant [10]. He reintroduced Poisson's azimuthal operator, $\mathscr{L}(\lambda)$, which allows the scattering term in Eq. (6) to be recast into

$$
\left.p_{\mathrm{sc}}^{(1)}\right|^{<}=-\left.\frac{2}{\pi} \int_{0}^{\rho} \frac{\mathrm{d} x}{\sqrt{\rho^{2}-x^{2}}} \int_{x}^{1} \frac{\rho_{0} \mathrm{~d} \rho_{0}}{\sqrt{\rho_{0}^{2}-x^{2}}} \mathscr{L}\left(\frac{x^{2}}{\rho \rho_{0}}\right) \frac{\partial p_{\text {pore }}}{\partial z}\right|^{<},
$$

provided that $\left.(\partial p / \partial z)\right|^{<}$belongs to $L^{1}[0,2 \pi]$ as a function of $\phi_{0}$ for any fixed $r \geqslant 0$. The details are recapitulated in Appendix A.

The integrals in Eq. (7) are in the form of Abel transform [11]. The complexity of solving Eq. (6) with Eq. (7) can be greatly reduced by applying their inverse operators,

$$
\mathscr{L}\left(\frac{1}{r}\right) \frac{\mathrm{d}}{\mathrm{d} r} \int_{0}^{r} \frac{\rho \mathrm{d} \rho}{\sqrt{r^{2}-\rho^{2}}} \mathscr{L}(\rho)
$$

and, subsequently,

$$
\mathscr{L}(t) \frac{\mathrm{d}}{\mathrm{d} t} \int_{t}^{1} \frac{r \mathrm{~d} r}{\sqrt{r^{2}-t^{2}}} \mathscr{L}\left(\frac{1}{r}\right)
$$


The details are relegated to Section B.1. The inversion procedures yield the unknown pressure derivative in terms of the pressure at the pore exit,

$$
\left.\frac{\pi t}{2} \frac{\partial p_{\text {pore }}^{(1)}}{\partial z}\right|^{<}=\frac{2 \mathrm{i} k_{x} t^{2} \cos \phi}{\sqrt{1-t^{2}}}+\left.\mathscr{L}(t) \frac{\mathrm{d}}{\mathrm{d} t} \int_{t}^{1} \frac{r \mathrm{~d} r}{\sqrt{r^{2}-t^{2}}} \mathscr{L}\left(\frac{1}{r^{2}}\right) \frac{\mathrm{d}}{\mathrm{d} r} \int_{0}^{r} \frac{\rho \mathrm{d} \rho}{\sqrt{r^{2}-\rho^{2}}} \mathscr{L}(\rho) p_{\text {pore }}^{(1)}\right|^{<}
$$

with $(t, \phi)$ as the radial and azimuthal coordinate variables.

The pressure in the pore region can be written as a linear combination of the eigenfunctions

$$
p_{\text {pore }}^{(1)}(\rho, \phi, z)=\sum_{m=0}^{\infty} \sum_{n=1}^{\infty} A_{m n}^{(1)} J_{m}\left(j_{m n}^{\prime} \rho\right) \cos (m \phi) \frac{\cosh \left[j_{m n}^{\prime}(z+\ell)\right]}{\cosh \left(j_{m n}^{\prime} \ell\right)},
$$

where $m$ is the azimuthal modal number and $j_{m n}^{\prime}$ is the $n$th zero of the derivative of the $m$ th order of the Bessel function of the first kind, i.e. $J_{m}^{\prime}\left(j_{m n}^{\prime}\right)=0$. The symmetry condition with respect to the $y=0$ plane leads to the sole dependence on the azimuthal factor $\cos (m \phi)$. The pressure and its pressure derivative at the pore exit are now

$$
\begin{aligned}
& \left.p_{\text {pore }}^{(1)}\right|^{<}=\sum_{m=0}^{\infty} \sum_{n=1}^{\infty} A_{m n}^{(1)} J_{m}\left(j_{m n}^{\prime} \rho\right) \cos (m \phi), \\
& \left.\frac{\partial p_{\text {pore }}^{(1)}}{\partial z}\right|^{<}=\sum_{m=0}^{\infty} \sum_{n=1}^{\infty} j_{m n}^{\prime} A_{m n}^{(1)} J_{m}\left(j_{m n}^{\prime} \rho\right) \cos (m \phi) \tanh \left(j_{m n}^{\prime} \ell\right) .
\end{aligned}
$$

In view of Eq. (10), it can be seen straightforwardly from the orthogonality of the cosine function that the non-vanishing components in the pore have a sole azimuthal mode $m=1$. Substituting Eqs. (11) and (12) and the definition of $\mathscr{L}(\lambda)$ operator into the integral equation (10), we have

$$
\begin{aligned}
& \frac{\pi t}{2} \sum_{n=1}^{\infty} j_{1 n}^{\prime} A_{1 n} \tanh \left(j_{1 n}^{\prime} \ell\right) J_{1}\left(j_{1 n}^{\prime} t\right) \\
& \quad=\frac{2 \mathrm{i} k_{x} t^{2}}{\sqrt{1-t^{2}}}-\sum_{n=1}^{\infty} \sqrt{\frac{\pi j_{1 n}^{\prime}}{2}} A_{1 n}^{(1)}\left\{j_{1 n}^{\prime} \int_{t}^{1} \frac{t^{2} \mathrm{~d} r}{\sqrt{r^{2}-t^{2}}} r^{-1 / 2} J_{3 / 2}\left(j_{1 n}^{\prime} r\right)+\frac{t^{2}}{\sqrt{1-t^{2}}} J_{1 / 2}\left(j_{1 n}^{\prime}\right)\right\}
\end{aligned}
$$

after carrying out the integrals and omitting the azimuthal factor $\cos \phi$.

Further taking operator $\int_{0}^{1} \mathrm{~d} t J_{1}\left(j_{1 l}^{\prime} t\right)(\cdot)$ in Eq. (13), we obtain an infinite system of algebraic equations for the unknown coefficients $A_{1 n}$

$$
\sum_{l=1}^{\infty} M_{n l}^{(1)} A_{1 l}^{(1)}=\frac{2 \mathrm{i} k_{x}}{j_{1 n}^{\prime 2}}\left(\sin j_{1 n}^{\prime}-j_{1 n}^{\prime} \cos j_{1 n}^{\prime}\right)
$$

with the help of formulas (5.54) and (6.567) of Ref. [12]. The matrix $M_{n l}^{(1)}$ is a symmetric array

$$
M_{n l}^{(1)}= \begin{cases}\frac{\pi}{2}\left\{\frac{j_{1 n}^{\prime}}{2}\left(J_{3 / 2}^{2}\left(j_{1 n}^{\prime}\right)-J_{1 / 2}\left(j_{1 n}^{\prime}\right) J_{5 / 2}\left(j_{1 n}^{\prime}\right)\right)+J_{3 / 2}\left(j_{1 n}^{\prime}\right) J_{1 / 2}\left(j_{1 n}^{\prime}\right)\right\} & \\ +\frac{\pi}{4} j_{1 n}^{\prime} \tanh \left(j_{1 n}^{\prime} \ell\right)\left(1-\frac{1}{j_{1 n}^{\prime}{ }^{2}}\right) J_{1}^{2}\left(j_{1 n}^{\prime}\right) & \text { if } n=l, \\ \frac{\pi\left(j_{1 n}^{\prime} j_{1 l}^{\prime}\right)^{-1 / 2}}{2 j_{1 n}^{\prime}{ }^{2}-j_{1 l}^{\prime 2}}\left\{j_{1 n}^{\prime}{ }^{2} j_{1 l}^{\prime} J_{3 / 2}\left(j_{1 n}^{\prime}\right) J_{1 / 2}\left(j_{1 l}^{\prime}\right)-j_{1 n}^{\prime} j_{1 l}^{\prime}{ }^{2} J_{1 / 2}\left(j_{1 n}^{\prime}\right) J_{3 / 2}\left(j_{1 l}^{\prime}\right)\right\} & \text { if } n \neq l .\end{cases}
$$

Eq. (14) can be solved easily without the need for any special numerical algorithms.

For the sake of further discussion, we denote the solution of the column vector of $A_{1 n}^{(1)}$ by

$$
\mathbf{A}_{1}^{(1)}=\mathrm{i} k_{x} \bar{A}_{1 n}^{(1)}
$$

Numerical calculation will be presented in Section 3.5. 


\subsection{Second-order inner solution}

The solution process of the second-order inner flow is similar to that discussed above. The total pressure for $z \geqslant 0$ of this order is

$$
p^{(2)}(\rho, \phi, z)=-\frac{1}{2}\left(k_{x}^{2} x^{2}+k_{z}^{2} z^{2}\right)+p_{\mathrm{sc}}^{(2)}(\rho, \phi, z) .
$$

The first term on the right-hand side is the external incident and reflected waves. Noting that its Laplacian automatically satisfies the source term of the Poisson equation (5), the scattering field, $p_{\mathrm{sc}}^{(2)}$ in $z \geqslant 0$, follows the Laplace equation, such that

$$
p_{\mathrm{sc}}^{(2)}(\rho, \phi, z)=-\left.\frac{1}{2 \pi} \int_{0}^{2 \pi} \int_{0}^{1} \frac{\partial p^{(2)}}{\partial z}\right|^{<} \frac{\rho_{0} \mathrm{~d} \rho_{0} \mathrm{~d} \phi_{0}}{\mathrm{~s}} .
$$

This leads to the integral equation for the sound pressure at the pore exit

$$
\left.p_{\text {pore }}^{(2)}\right|^{<}=-\frac{k_{x}^{2} x^{2}}{2}-\left.\frac{1}{2 \pi} \int_{0}^{2 \pi} \int_{0}^{1} \frac{\partial p_{\text {pore }}^{(2)}}{\partial z}\right|^{<} \frac{\rho_{0} \mathrm{~d} \rho_{0} \mathrm{~d} \phi_{0}}{\mathrm{~s}_{e}} .
$$

Applying the inverse operators and following the similar procedures in Section B.1, we inverse Eq. (18) to

$$
\begin{aligned}
\left.\frac{\pi t}{2} \frac{\partial p_{\text {pore }}^{(2)}}{\partial z}\right|^{<}= & -\frac{k_{x}^{2} t}{\sqrt{1-t^{2}}}\left(-\frac{1}{2}+t^{2}+\frac{2 t^{2}}{3} \cos (2 \phi)\right) \\
& +\left.\mathscr{L}(t) \frac{\mathrm{d}}{\mathrm{d} t} \int_{t}^{1} \frac{r \mathrm{~d} r}{\sqrt{r^{2}-t^{2}}} \mathscr{L}\left(\frac{1}{r^{2}}\right) \frac{\mathrm{d}}{\mathrm{d} r} \int_{0}^{r} \frac{\rho \mathrm{d} \rho}{\sqrt{r^{2}-\rho^{2}}} \mathscr{L}(\rho) p_{\text {pore }}^{(2)}\right|^{<} .
\end{aligned}
$$

Pressure in the pore region, satisfying Eq. (5) $)_{2}$, is

$$
p_{\text {pore }}^{(2)}(\rho, \phi, z)=A_{0}^{(2)}-\frac{1}{2}(z+\ell)^{2}+\sum_{m=0}^{\infty} \sum_{n=1}^{\infty} A_{m n}^{(2)} J_{m}\left(j_{m n}^{\prime} \rho\right) \cos (m \phi) \frac{\cosh \left[j_{m n}^{\prime}(z+\ell)\right]}{\cosh \left(j_{m n}^{\prime} \ell\right)} .
$$

The second term on the right-hand side is the particular solution to satisfy the source term due to the compressibility effect from the zeroth order and the rest are the homogeneous eigensolutions. Apparently, the non-zero components only exist for azimuthal modes $m=0$ and $m=2$. Substitution of Eq. (20) into Eq. (19) results in the respective equations for these two modes.

For $m=0$, the unknown coefficients can be solved by taking $\int_{0}^{1} \mathrm{~d} t(\cdot)$ and $\int_{0}^{1} \mathrm{~d} t J_{0}\left(j_{0 l}^{\prime} t\right)(\cdot)$ in Eq. (19). This leads to the system of equations

$$
\left(\begin{array}{cc}
1 & \mathbf{V}^{\mathrm{T}} \\
\mathbf{V} & { }_{0} \mathbf{M}^{(2)}
\end{array}\right)\left(\begin{array}{c}
A_{0}^{(2)} \\
\mathbf{A}_{0}^{(2)}
\end{array}\right)=\ell\left(\begin{array}{c}
\frac{\ell}{2}+\frac{\pi}{4} \\
\frac{\ell}{2} \mathbf{V}
\end{array}\right)-k_{x}^{2}\left(\begin{array}{c}
\frac{1}{6} \\
\mathbf{N}^{(2)}
\end{array}\right) .
$$

Symbols $\mathbf{A}_{0}^{(2)}, \mathbf{V}$ and $\mathbf{N}^{(2)}$ are column vectors:

$$
\begin{aligned}
& \mathbf{A}_{0}^{(2)}=A_{0 n}^{(2)}, \quad \mathbf{V}=V_{n}=\frac{\sin \left(j_{0 n}^{\prime}\right)}{j_{0 n}^{\prime}}, \\
& \mathbf{N}^{(2)}=N_{n}^{(2)}=\frac{\left(j_{0 n}^{\prime}{ }^{2}-2\right) \sin j_{0 n}^{\prime}+2 j_{0 n}^{\prime} \cos j_{0 n}^{\prime}}{2 j^{\prime 3}}
\end{aligned}
$$

and $\mathbf{V}^{\mathrm{T}}$ is the transpose of $\mathbf{V}$. The square submatrix, ${ }_{0} \mathbf{M}^{(2)}$, is

$$
{ }_{0} \mathbf{M}^{(2)}={ }_{0} M_{n l}^{(2)}= \begin{cases}\frac{1}{2}\left(1+\frac{\sin \left(2 j_{0 n}^{\prime}\right)}{2 j_{0 n}^{\prime}}\right)+\frac{\pi j_{0 n}^{\prime}}{4} \tanh \left(j_{0 n}^{\prime} \ell\right) J_{0}^{2}\left(j_{0 n}^{\prime}\right) & \text { if } n=l, \\ \frac{1}{2}\left(\frac{\sin \left(j_{0 n}^{\prime}-j_{0 l}^{\prime}\right)}{j_{0 n}^{\prime}-j_{0 l}^{\prime}}+\frac{\sin \left(j_{0 n}^{\prime}+j_{0 l}^{\prime}\right)}{j_{0 n}^{\prime}+j_{0 l}^{\prime}}\right) & \text { if } n \neq l .\end{cases}
$$


In view of Eq. (21), we can briefly express the solution of the coefficients

$$
\left(\begin{array}{c}
A_{0}^{(2)} \\
\mathbf{A}_{0}^{(2)}
\end{array}\right)=\ell\left(\begin{array}{c}
{ }_{0} \bar{A}_{0}^{(2)} \\
{ }_{0} \bar{A}_{0 n}^{(2)}
\end{array}\right)-k_{x}^{2}\left(\begin{array}{c}
{ }_{2} \bar{A}_{0}^{(2)} \\
{ }_{2} \bar{A}_{0 n}^{(2)}
\end{array}\right),
$$

where the first term contains the effect of the pore volume and the second accounts for the effect of the incident angle.

Similarly, taking the operator $\int_{0}^{1} \mathrm{~d} t J_{2}\left(j_{2 l}^{\prime} t\right)(\cdot)$ in Eq. (19) with Eq. (20), we obtain the system of algebraic equations for $m=2$,

$$
\sum_{l=1}^{\infty} M_{n l}^{(2)} A_{2 l}^{(2)}=-\frac{2 k_{x}^{2}}{3} \sqrt{\frac{\pi}{2 j_{2 n}^{\prime}}} J_{5 / 2}\left(j_{2 n}^{\prime}\right)
$$

and its solutions are

$$
A_{2 n}^{(2)}=-k_{x}^{2} \bar{A}_{2 n}^{(2)}
$$

The matrix ${ }_{2} \mathbf{M}^{(2)}$, equivalently, ${ }_{2} M_{n l}^{(2)}$, is

$$
{ }_{2} \mathbf{M}^{(2)}= \begin{cases}\frac{\pi}{2}\left\{\frac{j_{2 n}^{\prime}}{2}\left(J_{5 / 2}^{2}\left(j_{2 n}^{\prime}\right)-J_{3 / 2}\left(j_{2 n}^{\prime}\right) J_{7 / 2}\left(j_{2 n}^{\prime}\right)\right)+J_{3 / 2}\left(j_{2 n}^{\prime}\right) J_{5 / 2}\left(j_{2 n}^{\prime}\right)\right\} & \\ \quad+\frac{\pi}{4} j_{2 n}^{\prime} \tanh \left(j_{2 n}^{\prime} \ell\right)\left(1-\frac{4}{j_{2 n}^{\prime}{ }^{2}}\right) J_{2}^{2}\left(j_{2 n}^{\prime}\right) & \text { if } n=l, \\ \left.\frac{\pi\left(j_{2 n}^{\prime} j_{2 l}^{\prime}\right)^{-1 / 2}}{2} j_{2 n}^{\prime 2}-j_{2 l}^{\prime 2} j_{2 n}^{\prime}{ }^{2} j_{2 l}^{\prime} J_{5 / 2}\left(j_{2 n}^{\prime}\right) J_{3 / 2}\left(j_{2 l}^{\prime}\right)-j_{2 n}^{\prime} j_{2 l}^{\prime}{ }^{2} J_{3 / 2}\left(j_{2 n}^{\prime}\right) J_{5 / 2}\left(j_{2 l}^{\prime}\right)\right\} & \text { if } n \neq l .\end{cases}
$$

Both matrices ${ }_{0} \mathbf{M}^{(2)}$ and ${ }_{2} \mathbf{M}^{(2)}$ are symmetric.

\subsection{Far field of inner solution}

We present in this section only the principal procedures of the matching without emphasizing the rigorous details. One may verify the correctness of the results by following the routines, such as introducing an intermediate overlapping scale as in van Dyke [13], or using a simplified version by alternating expressions using the inner and outer variables as in Crighton [7].

The key point to match with the outer field is to express the far-field inner solution using the outer coordinate variables. The inner solution of the scattering pressure obtained in the previous section is, see Eqs. (6) and (17),

$$
\begin{aligned}
p_{\text {sc }}(\rho, \phi, z)= & -\frac{\varepsilon}{2 \pi s} \int_{0}^{2 \pi} \mathrm{d} \phi_{0} \int_{0}^{1} \rho_{0} \mathrm{~d} \rho_{0}\left(\left.\frac{\partial p_{\text {pore }}^{(1)}}{\partial z}\right|^{<}+\left.\varepsilon \frac{\partial p_{\text {pore }}^{(2)}}{\partial z}\right|^{<}\right) \\
& \times\left(1-\frac{2 \rho_{0} \sin \theta}{s} \cos \left(\phi-\phi_{0}\right)+\frac{\rho_{0}^{2}}{s^{2}}\right)^{-1 / 2},
\end{aligned}
$$

where $s^{2}=\rho^{2}+z^{2}$ is the distance from the origin to the observer at $(\rho, \phi, z)$, and $\theta$ is the colatitude angle between the position of the observer and the $z$ axis such that $\rho=s \sin \theta$.

For large $s$, the distance factor, the term in the second line of Eq. (25), can be expanded approximately

$$
\begin{gathered}
1+\frac{\rho_{0} \sin \theta}{s} \cos \left(\phi-\phi_{0}\right)+\frac{\rho_{0}^{2}}{2 s^{2}}\left(\frac{1}{2}-\frac{3}{2} \cos ^{2} \theta+\frac{3}{2} \sin ^{2} \theta \cos \left(2\left(\phi-\phi_{0}\right)\right)\right) \\
-\frac{3 \rho_{0}^{3}}{2 s^{3}}\left(\frac{\sin \theta}{4}\left(5 \cos ^{2} \theta-1\right) \cos \left(\phi-\phi_{0}\right)-\frac{5}{12} \sin ^{3} \theta \cos \left(3\left(\phi-\phi_{0}\right)\right)\right),
\end{gathered}
$$


using Taylor series with an accuracy up to $O\left(\left(\rho_{0} / s\right)^{4}\right)$. Maintaining this accuracy ensures that the match to the outer wave region is accurate up to $O\left(\varepsilon^{5}\right)$.

Because the leading pressure field, $p_{\text {pore }}^{(1)}$, Eq. (11), has only one azimuthal mode, $m=1$, with approximation (26), the only non-vanishing far-field terms from it are

$$
\begin{aligned}
- & \left.\frac{\varepsilon^{3}}{2 \pi S} \int_{0}^{2 \pi} \mathrm{d} \phi_{0} \int_{0}^{1} \mathrm{~d} \rho_{0} \rho_{0} \frac{\partial p^{(1)}}{\partial z}\right|^{<}\left\{\frac{\rho_{0} \sin \theta}{S} \cos \left(\phi-\phi_{0}\right)\right. \\
& \left.-\frac{3 \varepsilon^{2} \rho_{0}^{3}}{8 S^{3}} \sin \theta\left(5 \cos ^{2} \theta-1\right) \cos \left(\phi-\phi_{0}\right)\right\}
\end{aligned}
$$

where we have expressed $s$ in terms of the outer variable $S=|\mathbf{X}|=\varepsilon s$. The first integral is proportional to the first $x$-moment of the mass flux crossing the pore exit and the second term is one of the third moments of the flux, with the spherical mode $(3,1)$, cf. Section 3.4. With Eqs. (12) and (15), Eq. (27) can be integrated explicitly

$$
\begin{aligned}
- & \frac{\mathrm{i} k_{x} \varepsilon^{3}}{2 S^{2}} \sin \theta \cos \phi \sum_{n=1}^{\infty} \bar{A}_{1 n}^{(1)} \tanh \left(j_{1 n}^{\prime} \ell\right) J_{2}\left(j_{1 n}^{\prime}\right) \\
& +\frac{3 \mathrm{i} k_{x} \varepsilon^{5}}{S^{4}} \frac{\sin \theta}{16}\left(5 \cos ^{2} \theta-1\right) \cos \phi \sum_{n=1}^{\infty} \bar{A}_{1 n}^{(1)} \tanh \left(j_{1 n}^{\prime} \ell\right)\left(J_{2}\left(j_{1 n}^{\prime}\right)-\frac{2}{j_{1 n}^{\prime}} J_{3}\left(j_{1 n}^{\prime}\right)\right),
\end{aligned}
$$

where unique directivity patterns associated with each term are found.

The second-order inner solution has two azimuthal components, $m=0$ and $m=2$ of Eq. (20). For the $m=0$ mode, the far-field approximation is

$$
\frac{\varepsilon^{3}}{S} \int_{0}^{1} \mathrm{~d} \rho_{0} \rho_{0}\left(\ell-\sum_{n=1}^{\infty} j_{0 n}^{\prime} A_{0 n}^{(2)} \tanh \left(j_{0 n}^{\prime} \ell\right) J_{0}\left(j_{0 n}^{\prime} \rho_{0}\right)\right)\left(1+\frac{\varepsilon^{2} \rho_{0}^{2}}{2 S^{2}}\left(\frac{1}{2}-\frac{3}{2} \cos ^{2} \theta\right)\right)
$$

with the coefficients $A_{0 n}^{(2)}$ given by Eq. (22). The expression can be evaluated to

$$
\frac{\varepsilon^{3} \ell}{2 S}+\frac{\varepsilon^{5}}{2 S^{3}}\left(\frac{1}{2}-\frac{3}{2} \cos ^{2} \theta\right)\left(\frac{\ell}{4}+\sum_{n=1}^{\infty} \frac{2}{j_{0 n}^{\prime}} A_{0 n}^{(2)} \tanh \left(j_{0 n}^{\prime} \ell\right) J_{2}\left(j_{0 n}^{\prime}\right)\right) .
$$

The first term is physically the net total mass flux into the pore volume, and the other is the second moment $(2,0)$, cf. Section 3.4. It is worth noting that the leading term of Eq. (29) is $O\left(\varepsilon^{3}\right)$, and it is at the same order of that in Eq. (28). This term will be matched to the leading term of the scattering wave in the radiation field.

Finally, the far-field approximation for $m=2$ mode reads

$$
\frac{3 \varepsilon^{5}}{8 S^{3}} k_{x}^{2} \sin ^{2} \theta \cos (2 \phi) \sum_{n=1}^{\infty} \bar{A}_{2 n}^{(2)} \tanh \left(j_{2 n}^{\prime} \ell\right) J_{3}\left(j_{2 n}^{\prime}\right) .
$$

Eqs. (28)-(30) are to be matched with the outer wave region in the next subsection.

\subsection{Outer wave region}

The scattering wave in the outer region satisfies the governing equation $(2)_{2}$, the outgoing wave condition at infinity and the rigid wall at $Z=0$. From the expansions, (28)-(30), it is suggested that the scattering wave can be written in the form of

$$
P_{\mathrm{sc}}(S, \phi, \theta)=\varepsilon^{3} P_{\mathrm{sc}}^{(3)}(S, \phi, \theta)+\varepsilon^{5} P_{\mathrm{sc}}^{(5)}(S, \phi, \theta)+o\left(\varepsilon^{5}\right),
$$

where $(S, \phi, \theta)$ is the spherical coordinate position variables for $Z \geqslant 0$. From their directivity patterns, we conclude that $P^{(3)}$ and $P^{(5)}$ contain two and three multipoles, respectively. They are

$$
P_{\mathrm{sc}}^{(3)}(S, \phi, \theta)=B_{00}^{(3)} Y_{00}(\phi, \theta) h_{0}^{(1)}(S)+B_{11}^{(3)} Y_{11}(\phi, \theta) h_{1}^{(1)}(S),
$$




$$
\begin{aligned}
P_{\mathrm{sc}}^{(5)}(S, \phi, \theta)= & B_{20}^{(5)} Y_{20}(\phi, \theta) h_{2}^{(1)}(S)+B_{22}^{(5)} Y_{22}(\phi, \theta) h_{2}^{(1)}(S) \\
& +B_{31}^{(5)} Y_{31}(\phi, \theta) h_{3}^{(1)}(S),
\end{aligned}
$$

where $B_{m n}^{(3,5)}$ are the strengths of the multipoles yet to be determined. We use the combination $(m, n)$ to indicate the spherical modes and to distinguish the different types of multipoles. For example, $(0,0)$ is a monopole and $(1,0)$ and $(1,1)$ are the dipoles orienting in $z$ and $x$ directions. $Y_{m n}(\phi, \theta)$ are the real part of the spherical harmonic functions whose definitions are:

$$
\begin{aligned}
& Y_{00}=\frac{1}{\sqrt{4 \pi}}, \quad Y_{22}=\frac{1}{4} \sqrt{\frac{15}{2 \pi}} \sin ^{2} \theta \cos (2 \phi), \\
& Y_{11}=-\sqrt{\frac{3}{8 \pi}} \sin \theta \cos \phi, \quad Y_{31}=-\frac{1}{4} \sqrt{\frac{21}{4 \pi}} \sin \theta\left(5 \cos ^{2} \theta-1\right) \cos \phi \\
& Y_{20}=\sqrt{\frac{5}{4 \pi}}\left(\frac{3}{2} \cos ^{2} \theta-\frac{1}{2}\right),
\end{aligned}
$$

and $h_{m}^{(1)}(S)$ is the spherical Hankel function of the first kind such that

$$
h_{m}^{(1)}(S)=\sqrt{\frac{\pi}{2 S}}\left(J_{m+1 / 2}(S)+\mathrm{i} Y_{m+1 / 2}(S)\right)
$$

To match with the inner field of the wave components, we need the approximation of the spherical Hankel function for $S \rightarrow 0$ :

$$
\begin{array}{ll}
h_{0}^{(1)}(S)=-\frac{\mathrm{i}}{S}+1+\frac{\mathrm{i}}{2} S+O\left(S^{2}\right), & h_{2}^{(1)}(S)=-\frac{3 \mathrm{i}}{S^{3}}-\frac{\mathrm{i}}{2 S}+O(1), \\
h_{1}^{(1)}(S)=-\frac{\mathrm{i}}{S^{2}}-\frac{\mathrm{i}}{2}+\frac{S}{3}+O\left(S^{2}\right), & h_{3}^{(1)}(S)=-\frac{15 \mathrm{i}}{S^{4}}-\frac{3 \mathrm{i}}{2 S^{2}}+O(1) .
\end{array}
$$

Apart from the leading terms of each function in Eq. (35), the higher order terms only induce the flow field in the inner region of order higher than $\varepsilon^{3}$, which can be omitted in the present study. This can be seen by expressing Eq. (31) with Eq. (32), Eqs. (33) and (35) in terms of the inner variable $s$ and following the arguments in Ref. [7].

Therefore, the amplitude of the leading order multipoles of the scattering wave, Eq. (32), can be found by matching the coefficients of the leading terms of Eqs. (28) and (29) with the directivity (34) 1,3 . This yields

$$
B_{00}^{(3)}=\mathrm{i} \sqrt{\pi} \ell
$$

and

$$
B_{11}^{(3)}=-\sqrt{\frac{2 \pi}{3}} k_{x} \sum_{n=1}^{\infty} \bar{A}_{1 n}^{(1)} \tanh \left(j_{1 n}^{\prime} \ell\right) J_{2}\left(j_{1 n}^{\prime}\right)=-k_{x} \bar{B}_{11}^{(3)} .
$$

Eq. (36) represents that the strength of the monopole is linearly proportional to the depth of the pore and Eq. (37) is a dipole of $(1,1)$ induced by the oblique incident angle.

We further match the coefficients for the multipoles of $O\left(\varepsilon^{5}\right)$. The first term on the right-hand side of Eq. (33), matched with Eq. (29), is the quadrupole $(2,0)$. It contains the effects of both the pore volume and the incident wave,

$$
\begin{aligned}
B_{20}^{(5)} & =-\frac{\mathrm{i}}{3} \sqrt{\frac{\pi}{5}}\left(\frac{\ell}{4}+\sum_{n=1}^{\infty} \frac{2}{j_{0 n}^{\prime}}\left(\ell_{0} \bar{A}_{0 n}^{(2)}-k_{x_{2}}^{2} \bar{A}_{0 n}^{(2)}\right) \tanh \left(j_{0 n}^{\prime} \ell\right) J_{2}\left(j_{0 n}^{\prime}\right)\right) \\
& =-\frac{\mathrm{i}}{12} \sqrt{\frac{\pi}{5}} \ell-\mathrm{i} \ell_{0} \bar{B}_{20}^{(5)}+\mathrm{i} k_{x_{2}}^{2} \bar{B}_{20}^{(5)}
\end{aligned}
$$


with the coefficients $A_{0 n}^{(2)}$, Eq. (22), expanded. The second term, with Eq. (24), is the quadrupole (2, 2), which is purely induced by the oblique incident wave

$$
B_{22}^{(5)}=\frac{\mathrm{i}}{2} \sqrt{\frac{2 \pi}{15}} k_{x}^{2} \sum_{n=1}^{\infty} \bar{A}_{2 n}^{(2)} \tanh \left(j_{2 n}^{\prime} \ell\right) J_{3}\left(j_{2 n}^{\prime}\right)=\mathrm{i} k_{x}^{2} \bar{B}_{22}^{(5)} .
$$

The last term is then the octupole $(3,1)$ :

$$
B_{31}^{(5)}=\frac{k_{x}}{10} \sqrt{\frac{\pi}{21}} \sum_{n=1}^{\infty} \bar{A}_{1 n}^{(1)} \tanh \left(j_{1 n}^{\prime} \ell\right)\left(J_{2}\left(j_{1 n}^{\prime}\right)-\frac{2}{j_{1 n}^{\prime}} J_{3}\left(j_{1 n}^{\prime}\right)\right)=k_{x} \bar{B}_{31}^{(5)} .
$$

Equations from (36) to (40) are the effective moments of the multipoles in the present scattering problem. Their dependence on the pore depth and incident wave angle is explicitly shown in the expressions. The moment coefficients, $\bar{B}_{00}^{(3)}, \bar{B}_{11}^{(3)}, \bar{B}_{20}^{(5)}, \bar{B}_{22}^{(5)}$ and $\bar{B}_{31}^{(5)}$ are defined to simplify the following discussion, and they are dependent only on the pore depth.

\subsection{Calculation and discussion}

We solve the multipoles of the scattering wave in the last section and obtain three infinite systems of algebraic equations, (14), (21) and (23), for the inner flows. For numerical illustration, we truncate the systems into finite sizes provided that a good match at the pore exit is ensured. The convergence with the number of terms, $N$, is plotted in Fig. 2(a). The representative error for each $N$ is the maximum error in the total pressure, taken from $x=0$ to 0.97 . The error occurs near the exit corner because it is a geometric singular point. We use $N=150$ for the monopole and 120 for the others. They are verified to produce an absolute error less than about 0.015 and a relative error less than $3 \%$ at the exit. Fig. 2(b) shows $\bar{A}_{1 n}^{(1)}$ for $\ell=1.5$, a typical serial of the unknowns of the equation sets. It converges to zero with alternating signs.

The three equation sets are associated with three different inner flow fields, as sketched in Figs. 3-5. The first-order inner flow is an incompressible field with an external uniform flow parallel to the flanged surface, Eq. (6). The normalized total pressure, $-\mathrm{i} p^{(1)} / k_{x}$, gives a base flow field shown in Fig. 3(a). This flow vanishes when the wave is normally incident on the pore and reaches the maximum when the incident angle approaches $90^{\circ}$. The modification due to the pore on the mean flow, Fig. 3(b), is an incompressible dipole, or doublet, with a strength proportional to the $x$-moment of the mass flux entering/leaving the pore, seen from the first term of Eq. (27). It has a directivity pattern of a two-lobed structure. We omit the duct field because of discontinuity in pressure after subtracting the external flow.

The second-order inner flow is under the influence of both the fluid compressibility and the incident angle. The small but non-negligible compressibility allows the flow to be compressed slightly during the compressing phase. This causes extra mass to be stored in/near the pore volume. When the external pressure releases, the

(a)

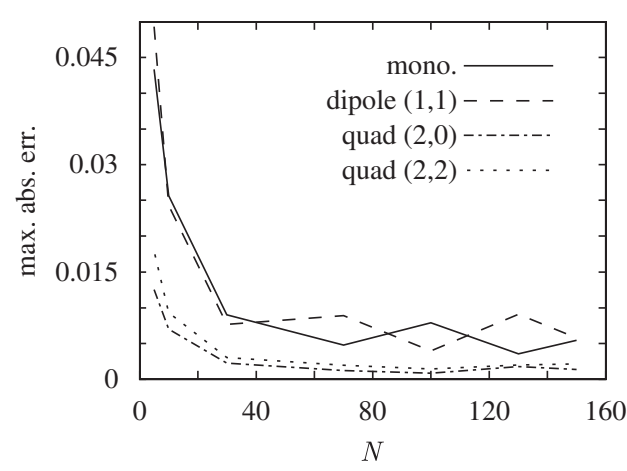

(b)

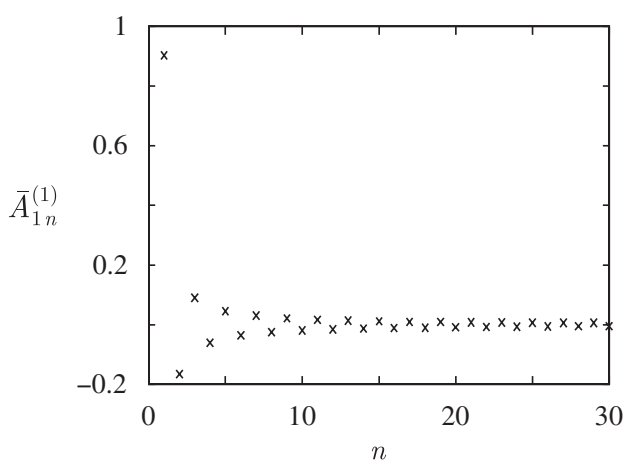

Fig. 2. (a) Absolute errors at the exit versus $N$ and (b) $\bar{A}_{1 n}^{(1)}$ for $\ell=1.5$. 
(a)

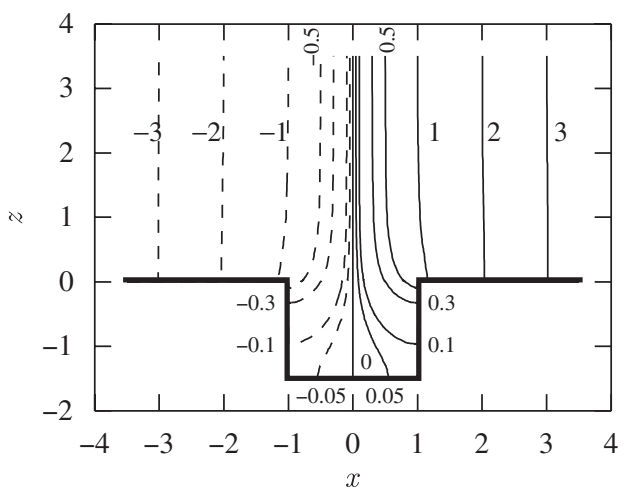

(b)

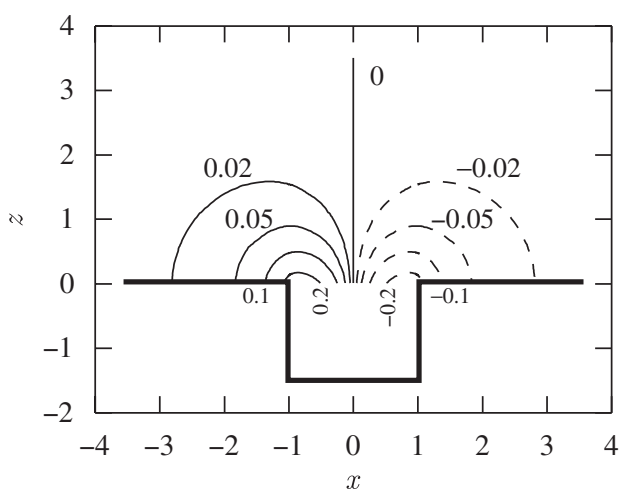

Fig. 3. Pressure contour of the inner field for $\ell=1.5$ for (a) total pressure, $-\mathrm{i} p^{(1)} / k_{x}$, (b) doublet $(1,1),-\mathrm{i} p^{(1)} / k_{x}-x$ for $z \geqslant 0$.

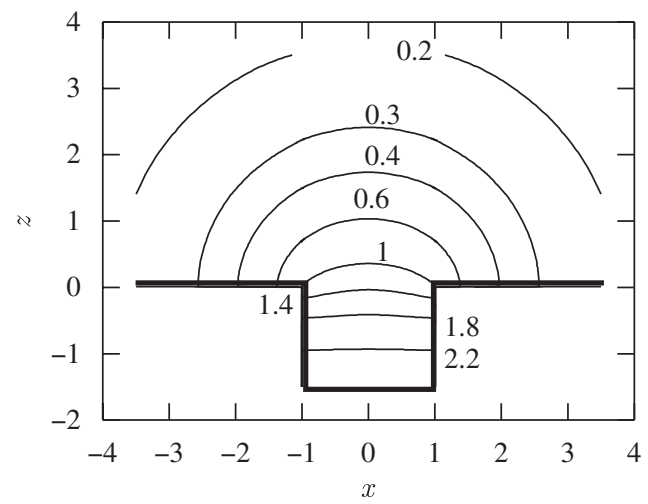

Fig. 4. Pressure contour, $p^{(2)} / \ell$, of the inner monopole for $\ell=1.5$.

(a)

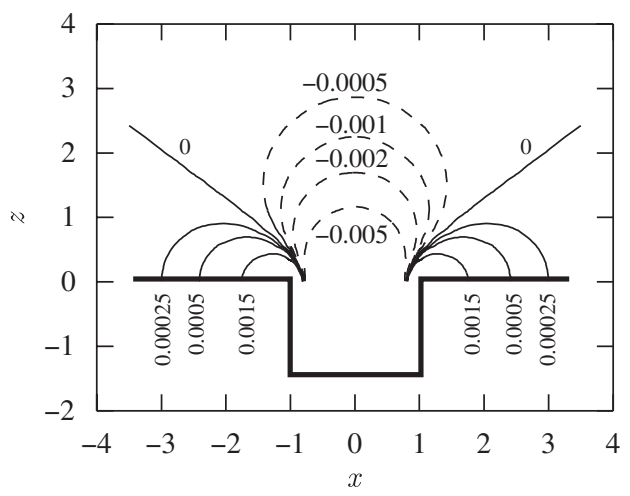

(b)

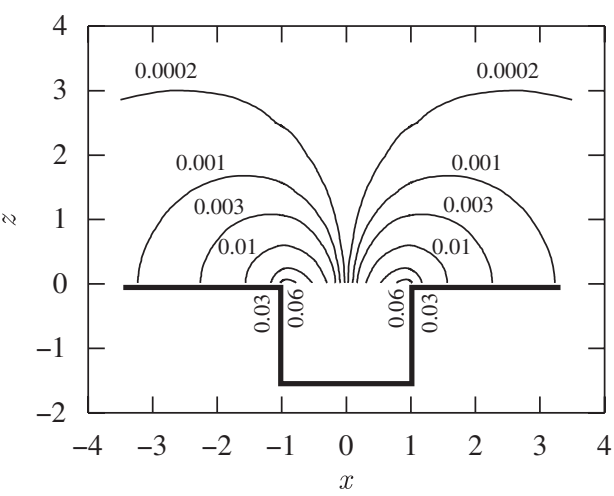

Fig. 5. Incident angle-induced pressure contour, $\left.k_{x}^{-2} p^{(2)}\right|_{\ell=0}+x^{2} / 4$ for $z \geqslant 0$, of the inner quadrulet field for $\ell=1.5$ : (a) quadrulet (2,0) and (b) quadrulet $(2,2)$.

mass is discharged from the pore and becomes a monopole source. This is seen from the first two terms of the duct solution, (20). The monopole flow is shown in Fig. 4. The strength of the monopole is linearly proportional to the storage capability of the pore, i.e. the depth see the first term of Eq. (29). On the other hand, there are two flow fields in this order caused by the incident angle. They have azimuthal 
modes $m=0$ and $m=2$, which are induced from the incident pressure, $-k_{x}^{2} \rho^{2} \cos ^{2} \phi / 2$, at the pore exit, Eq. (16). The induced flows have two quadrupoles, or quadrulets, $(2,0)$ and $(2,2)$, shown in Fig. 5(a,b). The quadrupole flows in the figure are normalized by $k_{x}^{2}$.

The far-field scattering fields are given by the matching procedures and the results are tabulated in Table 1 . The leading terms, Eq. (32), are of $O\left(\varepsilon^{3}\right)$ and contain one monopole and one dipole. The monopole arises from the second-order inner field and becomes the leading order of the wave field because of its slower spatial decay rate, as pointed out in Ref. [7]. The dipole is induced from the incident angle of the external wave. After extracting the angle dependence, its moment coefficient, $\bar{B}_{11}^{(3)}$, is a function which depends only on the depth, as shown in Fig. 6(a). The dipole has an accompanying higher order term, which is the octupole $(3,1)$ of $O\left(\varepsilon^{5}\right)$ resulting from the second term of Eq. (28). The moment coefficient $\bar{B}_{31}^{(5)}$ is calculated and plotted in Fig. 6(b). Both of the moment coefficients vanish when $\ell \rightarrow 0$ and saturate around $\ell \approx 1$.

The next order of magnitude of the scattering wave is $O\left(\varepsilon^{5}\right)$. In addition to the mentioned octupole, it further consists of two quadrupoles: $(2,0)$ and $(2,2)$. The quadrupole $(2,0)$ has the most complicated structure

Table 1

Multipole structures for the rigid surface

\begin{tabular}{lll}
\hline Multipole & $O\left(\varepsilon^{3}\right)$ & $O\left(\varepsilon^{5}\right)$ \\
\hline Monopole & $\mathrm{i} \ell \sqrt{\pi}$ & \\
Dipole (1,1) & $-k_{x} \bar{B}_{11}^{(3)}$ & \\
Quadrupole (2,0) & & $\mathrm{i}$ \\
& & $\sqrt{\frac{\pi}{5}} \ell-\mathrm{i} \ell_{0} \bar{B}_{20}^{(5)}+k_{x 2}^{2} \bar{B}_{20}^{(5)}$ \\
Quadrupole (2,2) & & $\mathrm{i} k_{x}^{2} \bar{B}_{22}^{(5)}$ \\
Octupole $(3,1)$ & $k_{x} \bar{B}_{31}^{(5)}$ \\
\hline
\end{tabular}

(a)

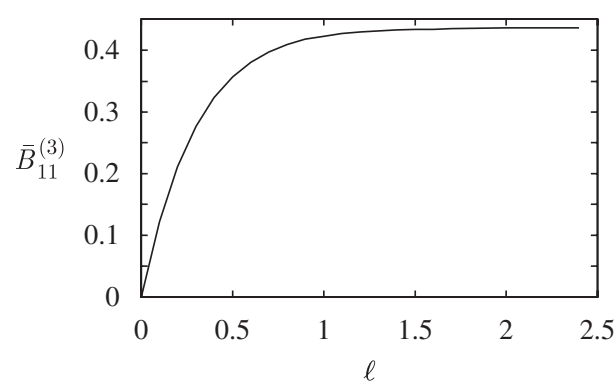

(b)

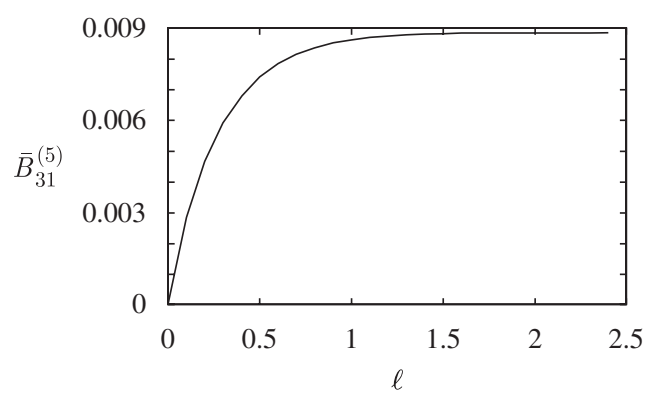

Fig. 6. The effective multipole moment coefficient versus pore depth of (a) dipole (2,1), Eq. (37) and (b) octupole (3,1), Eq. (40).

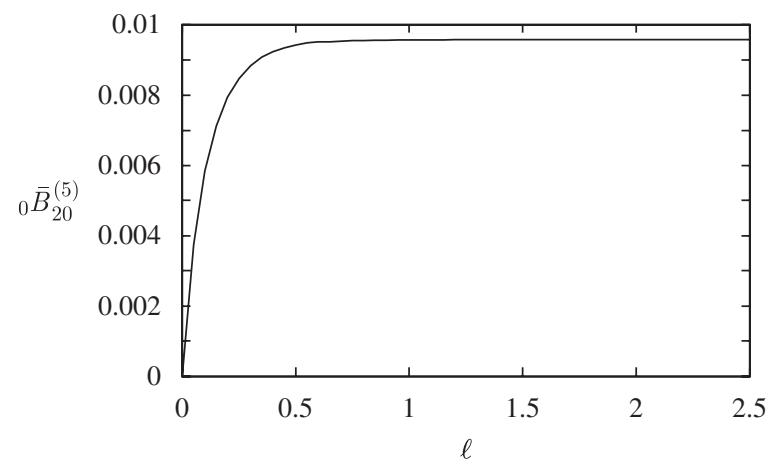

Fig. 7. The effective moment coefficient of quadrupole $(2,0),{ }_{0} \bar{B}_{20}^{(5)}$ of Eq. (38). 
(a)

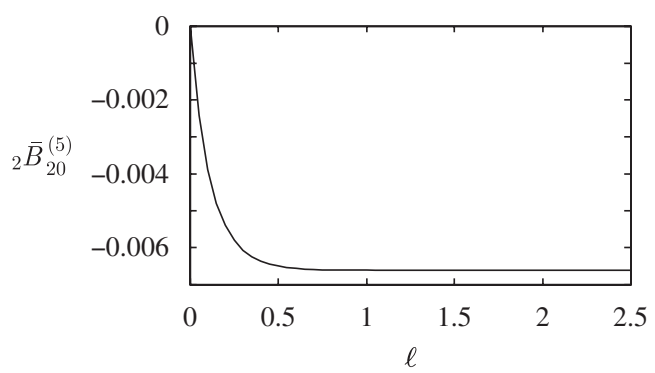

(b)

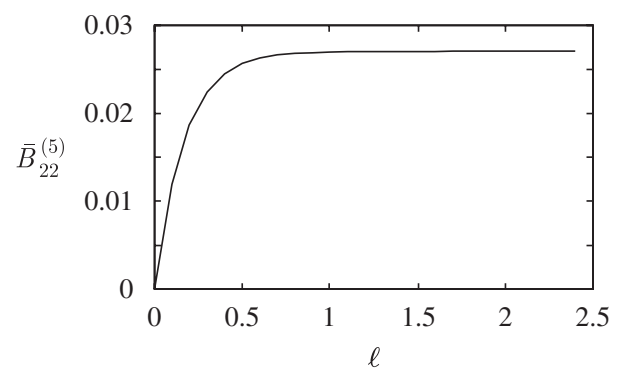

Fig. 8. The effective multipole moment coefficient versus pore depth of (a) quadrupole $(2,0),{ }_{2} \bar{B}_{20}^{(5)}$ of Eq. (38) and (b) quadrupole (2,2), Eq. (39).

and is under the influence of both the fluid compressibility and the incident angle, Eq. (38). The term scaling linearly with $\ell$ is due to the non-vanishing second moment of the compressed mass flowing into the volume. Its moment coefficient, ${ }_{0} \bar{B}_{20}^{(5)}$, is sketched in Fig. 7. The non-zero incident angle raises the remaining terms of the quadrupoles $(2,0)$ and $(2,2)$. Their moment coefficients, ${ }_{2} \bar{B}_{20}^{(5)}$ and $\bar{B}_{22}^{(5)}$, are shown in Figs. 8(a) and (b), respectively.

\section{Pressure-release surface}

\subsection{Inner flow}

As argued in the introduction, the solution for the pressure-release condition is valuable when extending the current solution to systems such as electro-magnetic waves, regardless of whether there are any real materials providing such a condition acoustically. The pressure-release condition gives $p(\mathbf{x})=0$ on the boundary surface.

As in Section 3, we carry out the inner flow up to a second-order accuracy. The external incident and reflected waves over a pressure-release surface at $z=0$ are:

$$
p_{\text {ext }}=-\mathrm{i} \varepsilon k_{z} z+\varepsilon^{2} k_{x} k_{z} x z+O\left(\varepsilon^{3}\right)
$$

after normalizing by $2 I^{*}$. We denote $p_{\mathrm{ext}}^{(1)}=-\mathrm{i} k_{z} z$ and $p_{\mathrm{ext}}^{(2)}=k_{x} k_{z} x z$ in the following derivation. Their derivatives with respect to $z$ are $-\mathrm{i} k_{z}$ and $k_{x} k_{z} x=k_{x} k_{z} \rho \cos \phi$, respectively. This expansion indicates that the total pressure expansion of the inner region is $p(\mathbf{x})=\varepsilon p^{(1)}(\mathbf{x})+\varepsilon^{2} p^{(2)}(\mathbf{x})$ and, subsequently, the pressures, $p^{(1)}$ and $p^{(2)}$, satisfy the Laplace equations: see Eq. (2) $)_{1}$.

The non-dimensionalized total pressures in the inner flow region can be written down straightforwardly using the same Green function in Section 3.1:

$$
p^{(1,2)}(\mathbf{x})=p_{\text {ext }}^{(1,2)}(\mathbf{x})-\left.\frac{1}{2 \pi} \int_{0}^{2 \pi} \int_{0}^{1} \frac{\partial p_{\mathrm{sc}}^{(1,2)}}{\partial z}\right|^{<} \frac{\rho_{0} \mathrm{~d} \rho_{0} \mathrm{~d} \phi_{0}}{\mathrm{~s}}-\left.\frac{1}{2 \pi} \int_{0}^{2 \pi} \int_{1}^{\infty} \frac{\partial p_{\mathrm{sc}}^{(1,2)}}{\partial z}\right|^{>} \frac{\rho_{0} \mathrm{~d} \rho_{0} \mathrm{~d} \phi_{0}}{\mathrm{~s}} .
$$

The evaluation symbol, $\left.\right|^{>}$, indicates that the operand is evaluated in the region $\rho \geqslant 1$ at $z=0$ or $\rho_{0} \geqslant 1$ at $z_{0}=0$, whichever is appropriate. This is a summation of the external wave field, $p_{\text {ext }}^{(1,2)}(\mathbf{x})$, and the scattering waves due to the pore. The superscript ${ }^{(1,2)}$ corresponds to the physical quantities of the first or second order.

Both $\left.\left(\partial p_{\mathrm{sc}}^{(1,2)} / \partial z\right)\right|^{<,>}$are unknowns at the moment. For the pressure-release condition, the pressure vanishes on the flanged surface. Utilizing this condition, we bring the observation point $\mathbf{x}$ in Eq. (41) to the flanged surface. This gives an integral equation

$$
\left.\int_{0}^{2 \pi} \int_{1}^{\infty} \frac{\partial p_{\mathrm{sc}}^{(1,2)}}{\partial z}\right|^{>} \frac{\rho_{0} \mathrm{~d} \rho_{0} \mathrm{~d} \phi_{0}}{\mathrm{~s}_{e}}=-\left.\int_{0}^{2 \pi} \int_{0}^{1} \frac{\partial p_{\mathrm{sc}}^{(1,2)}}{\partial z}\right|^{<} \frac{\rho_{0} \mathrm{~d} \rho_{0} \mathrm{~d} \phi_{0}}{\mathrm{~s}_{e}}
$$


for $\rho \geqslant 1$ at $z=0$. Applying the azimuthal operator $\mathscr{L}(\cdot)$, with details in Section B.2, we recast Eq. (42) into

$$
\begin{aligned}
& \left.\int_{\rho}^{\infty} \frac{\mathrm{d} x}{\sqrt{x^{2}-\rho^{2}}} \int_{1}^{x} \frac{\rho_{0} \mathrm{~d} \rho_{0}}{\sqrt{x^{2}-\rho_{0}^{2}}} \mathscr{L}\left(\frac{\rho \rho_{0}}{x^{2}}\right) \frac{\partial p_{\mathrm{sc}}^{(1,2)}}{\partial z}\right|^{>} \\
& \quad=-\left.\int_{\rho}^{\infty} \frac{\mathrm{d} x}{\sqrt{x^{2}-\rho^{2}}} \int_{0}^{1} \frac{\rho_{0} \mathrm{~d} \rho_{0}}{\sqrt{x^{2}-\rho_{0}^{2}}} \mathscr{L}\left(\frac{\rho \rho_{0}}{x^{2}}\right) \frac{\partial p_{\mathrm{sc}}^{(1,2)}}{\partial z}\right|^{<},
\end{aligned}
$$

which is ready to be inverted.

The sequence of the inverse operators is

$$
\mathscr{L}(t) \frac{\mathrm{d}}{\mathrm{d} t} \int_{t}^{\infty} \frac{\rho \mathrm{d} \rho}{\sqrt{\rho^{2}-t^{2}}} \mathscr{L}\left(\frac{1}{\rho}\right) \text { and } \mathscr{L}\left(\frac{1}{\rho}\right) \frac{\mathrm{d}}{\mathrm{d} \rho} \int_{1}^{\rho} \frac{t \mathrm{~d} t}{\sqrt{\rho^{2}-t^{2}}} \mathscr{L}(t)
$$

as proposed in Ref. [10]. After taking the inverse operators, we obtain the pressure derivative on the flanged surface in terms of that at the pore exit, e.g.

$$
\left.\frac{\partial p_{\mathrm{sc}}^{(1,2)}}{\partial z}\right|^{>}=-\left.\frac{2}{\pi \sqrt{\rho^{2}-1}} \int_{0}^{1} \frac{\sqrt{1-\rho_{0}^{2}} \rho_{0} \mathrm{~d} \rho_{0}}{\rho^{2}-\rho_{0}^{2}} \mathscr{L}\left(\frac{\rho_{0}}{\rho}\right) \frac{\partial p_{\mathrm{sc}}^{(1,2)}}{\partial z}\right|^{<} .
$$

In order to match the pressure condition with the pore solution, it is more convenient to recast $\left.\left(\partial p_{\mathrm{sc}}^{(1,2)} / \partial z\right)\right|^{>}$in terms of $\left.\left(\partial p_{\mathrm{sc}}^{(1,2)} / \partial z\right)\right|^{<}$. For this purpose, we again take $\mathbf{x}$ in Eq. (41) to the pore exit area and substitute with Eq. (45). We also have the continuity of the pressure and the pressure derivative at the pore exit: $\left.p^{(1,2)}\right|^{<}=\left.p_{\text {pore }}^{(1,2)}\right|^{<}$, and $\left.\left(\partial p_{\mathrm{sc}}^{(1,2)} / \partial z\right)\right|^{<}=-\left.\left(\partial p_{\text {ext }}^{(1,2)} / \partial z\right)\right|^{<}+\left.\left(\partial p_{\text {pore }}^{(1,2)} / \partial z\right)\right|^{<}$, respectively. With the details in Section B.3, we have

$$
\left.p_{\text {pore }}^{(1,2)}\right|^{<}=-\frac{2}{\pi} \int_{\rho}^{1} \frac{\mathrm{d} x}{\sqrt{x^{2}-\rho^{2}}} \int_{0}^{x} \frac{\rho_{0} \mathrm{~d} \rho_{0}}{\sqrt{x^{2}-\rho_{0}^{2}}} \mathscr{L}\left(\frac{\rho \rho_{0}}{x^{2}}\right)\left(-\left.\frac{\partial p_{\text {ext }}^{(1,2)}}{\partial z}\right|^{<}+\left.\frac{\partial p_{\text {pore }}^{(1,2)}}{\partial z}\right|^{<}\right) .
$$

Eq. (46) is the equivalent integral equation for the pressure-release condition, but now uses the pore pressure as the unknown.

Pressure field in the pore region can be expanded by the eigensolutions

$$
p_{\text {pore }}^{(1,2)}(\rho, \phi, z)=\sum_{m=0}^{\infty} \sum_{n=1}^{\infty} \frac{A_{m n}^{(1,2)}}{j_{m n}} J_{m}\left(j_{m n} \rho\right) \cos (m \phi) \frac{\sinh \left[j_{m n}(z+\ell)\right]}{\cosh \left(j_{m n} \ell\right)},
$$

where the constant $j_{m n}$ satisfies $J_{m}\left(j_{m n}\right)=0$. This form is chosen particularly so that symmetric algebraic systems are achieved later. At $z=0$, the pressure and its derivative are:

$$
\begin{aligned}
& \left.p_{\text {pore }}^{(1,2)}\right|^{<}=\sum_{m=0}^{\infty} \sum_{n=1}^{\infty} \frac{A_{m n}^{(1,2)}}{j_{m n}} \tanh \left(j_{m n} \ell\right) J_{m}\left(j_{m n} \rho\right) \cos (m \phi), \\
& \left.\frac{\partial p_{\text {pore }}^{(1,2)}}{\partial z}\right|^{<}=\sum_{m=0}^{\infty} \sum_{n=1}^{\infty} A_{m n}^{(1,2)} J_{m}\left(j_{m n} \rho\right) \cos (m \phi) .
\end{aligned}
$$

Substituting Eqs. (47) and (48) into Eq. (46), we have

$$
\begin{aligned}
& \sum_{m=0}^{\infty} \sum_{n=1}^{\infty} \frac{A_{m n}^{(1,2)}}{j_{m n}} \tanh \left(j_{m n} \ell\right) J_{m}\left(j_{m n} \rho\right) \cos (m \phi)=-\frac{2}{\pi} \int_{\rho}^{1} \frac{\mathrm{d} x}{\sqrt{x^{2}-\rho^{2}}} \int_{0}^{x} \frac{\rho_{0} \mathrm{~d} \rho_{0}}{\sqrt{x^{2}-\rho_{0}^{2}}} \\
& \quad \times \mathscr{L}\left(\frac{\rho \rho_{0}}{x^{2}}\right)\left(-\left.\frac{\partial p_{\mathrm{ext}}^{(1,2)}}{\partial z}\right|^{<}+\sum_{m=0}^{\infty} \sum_{n=1}^{\infty} A_{m n}^{(1,2)} J_{m}\left(j_{m n} \rho_{0}\right) \cos (m \phi)\right) .
\end{aligned}
$$

From the orthogonality of the cosine functions, the only non-vanishing azimuthal modes for the first- and second-order inner flow are $m=0$ and 1 , respectively. Taking $\int_{0}^{1} \mathrm{~d} \rho \rho J_{0}\left(j_{0 l} \rho\right)(\cdot)$ and $\int_{0}^{1} \mathrm{~d} \rho \rho J_{1}\left(j_{1 l} \rho\right)(\cdot)$, we can 
rewrite Eq. (49) into two infinite algebraic equation systems:

$$
\mathbf{M}^{(1)} \mathbf{A}_{0}^{(1)}=-\mathrm{i} k_{z} \mathbf{N}^{(1)}, \quad \mathbf{M}^{(2)} \mathbf{A}_{1}^{(2)}=k_{x} k_{z} \mathbf{N}^{(2)},
$$

for the two orders of the inner flow. Their solutions are abbreviated as

$$
\begin{aligned}
& \mathbf{A}_{0}^{(1)}=-\mathrm{i} k_{z}\left(\mathbf{M}^{(1)}\right)^{-1} \mathbf{N}^{(1)}=-\mathrm{i} k_{z} \bar{A}_{0 n}^{(1)}, \\
& \mathbf{A}_{1}^{(2)}=k_{x} k_{z}\left(\mathbf{M}^{(2)}\right)^{-1} \mathbf{N}^{(2)}=k_{x} k_{z} \bar{A}_{1 n}^{(2)} .
\end{aligned}
$$

The matrices and vectors in Eq. (50) are explicitly

$$
\begin{aligned}
& \mathbf{N}^{(1)}= \sqrt{\frac{2}{\pi} j_{0 n}^{-3 / 2} J_{3 / 2}\left(j_{0 n}\right),} \\
& \mathbf{M}^{(1)}= \begin{cases}-\frac{1}{2 j_{0 n}} \tanh \left(j_{0 n} \ell\right) J_{-1}\left(j_{0 n}\right) J_{1}\left(j_{0 n}\right) & \text { if } n=l, \\
\quad+\frac{1}{2 j_{0 n}}\left\{J_{1 / 2}^{2}\left(j_{0 n}\right)-J_{-1 / 2}\left(j_{0 n}\right) J_{3 / 2}\left(j_{0 n}\right)\right\} & \\
\frac{\left(j_{0 n} j_{0 l}\right)^{-1 / 2}}{j_{0 n}^{2}-j_{0 l}^{2}}\left\{j_{0 l} J_{1 / 2}\left(j_{0 n}\right) J_{-1 / 2}\left(j_{0 l}\right)-j_{0 n} J_{-1 / 2}\left(j_{0 n}\right) J_{1 / 2}\left(j_{0 l}\right)\right\} & \text { if } n \neq l\end{cases}
\end{aligned}
$$

and

$$
\begin{aligned}
& \mathbf{N}^{(2)}= \frac{2}{3} \sqrt{\frac{2}{\pi} j_{1 n}^{-3 / 2} J_{5 / 2}\left(j_{1 n}\right),} \\
& \mathbf{M}^{(2)}= \begin{cases}-\frac{1}{2 j_{1 n}} \tanh \left(j_{1 n} \ell\right) J_{0}\left(j_{1 n}\right) J_{2}\left(j_{1 n}\right) & \\
+\frac{1}{2 j_{1 n}}\left\{J_{3 / 2}^{2}\left(j_{1 n}\right)-J_{1 / 2}\left(j_{1 n}\right) J_{5 / 2}\left(j_{1 n}\right)\right\} & \text { if } n=l, \\
\frac{\left(j_{1 n} j_{1 l}\right)^{-1 / 2}}{j_{1 n}^{2}-j_{1 l}^{2}}\left\{j_{1 l} J_{3 / 2}\left(j_{1 n}\right) J_{1 / 2}\left(j_{1 l}\right)-j_{1 n} J_{1 / 2}\left(j_{1 n}\right) J_{3 / 2}\left(j_{1 l}\right)\right\} & \text { if } n \neq l .\end{cases}
\end{aligned}
$$

Matrices $\mathbf{M}^{(1,2)}$ are confirmed symmetric.

\subsection{Outer wave region}

In Section 4.1, the inner pressure fields for the leading two orders of magnitude are solved. Following the same principle of the method of the matched asymptotic expansion, the outer wave field is to be determined from the far-field behavior of the inner pressure, i.e. by letting $s \rightarrow \infty$ in Eq. (41). However, since the integration domain of the second integral of Eq. (41) extends to infinity, this introduces unnecessary complications in the calculation while taking the limit of $\mathbf{s}$ at small $z$. This can be avoided if we reformulate the scattering pressure in an alternative expression,

$$
p_{\mathrm{sc}}^{(1,2)}(\rho, \phi, z)=\int_{0}^{2 \pi} \int_{0}^{1}\left(\left.\left.p_{\mathrm{sc}}^{(1,2)}\right|^{<} \frac{\partial G\left(\rho, \phi, z ; \rho_{0}, \phi_{0}, z_{0}\right)}{\partial z_{0}}\right|_{z_{0}=0}\right) \rho_{0} \mathrm{~d} \rho_{0} \phi_{0}
$$

with the semi-infinite Green function $G\left(\rho, \phi, z ; \rho_{0}, \phi_{0}, z_{0}\right)$ satisfying $\left.G\right|_{z_{0}=0}=0$ at $z=0$. The derivative of $G$ at $z_{0}=0$ is, therefore,

$$
\left.\frac{\partial G}{\partial z_{0}}\right|_{z_{0}=0}=\frac{z}{2 \pi \mathbf{s}^{3}}
$$


by the method of image after taking $z_{0} \rightarrow 0$. The far-field approximation of (52), as $s=\sqrt{\rho^{2}+z^{2}} \rightarrow \infty$, is

$$
\frac{\cos \theta}{2 \pi s^{2}}\left\{1+\frac{3 \rho_{0}}{s} \sin \theta \cos \left(\phi-\phi_{0}\right)-\frac{3 \rho_{0}^{2}}{2 s^{2}}\left(\frac{5}{2} \cos ^{2} \theta-\frac{3}{2}\right)+\frac{15 \rho_{0}^{2}}{4 s^{2}} \sin ^{2} \theta \cos \left(2\left(\phi-\phi_{0}\right)\right)\right\},
$$

where $\theta$ has the same definition as that in Section 3.3, i.e. $\rho=s \sin \theta$. Following the process outlined in Sections 3.3 and 3.4, we have the outer wave field in the same form of Eq. (31), but with a different multipole expansion structure,

$$
\begin{aligned}
& P_{\mathrm{sc}}^{(3)}(S, \phi, \theta)=B_{10}^{(3)} Y_{10}(\phi, \theta) h_{1}^{(1)}(S), \\
& P_{\mathrm{sc}}^{(5)}(S, \phi, \theta)=B_{21}^{(5)} Y_{21}(\phi, \theta) h_{2}^{(1)}(S)+B_{30}^{(5)} Y_{30}(\phi, \theta) h_{3}^{(1)}(S) .
\end{aligned}
$$

The directivities are given by the spherical harmonics

$$
\begin{aligned}
& Y_{10}(\rho, \phi)=\frac{1}{2} \sqrt{\frac{3}{\pi}} \cos \theta, \quad Y_{30}(\rho, \phi)=\sqrt{\frac{7}{16 \pi}}\left(5 \cos ^{3} \theta-3 \cos \theta\right) . \\
& Y_{21}(\rho, \phi)=-\sqrt{\frac{15}{8 \pi}} \sin \theta \cos \theta \cos \phi,
\end{aligned}
$$

The moments $B_{10}^{(3)}, B_{21}^{(5)}$ and $B_{30}^{(5)}$ are simply proportional to the integrals $\left.\int p_{\mathrm{sc}}\right|^{<} \mathrm{d} A,\left.\int x p_{\mathrm{sc}}\right|^{<} \mathrm{d} A$ and $\int \rho_{0}^{2} p_{\mathrm{sc}}<\mathrm{d} A$ with the area element $\mathrm{d} A$ of the pore exit. Contrary to the case of the rigid boundary condition, these integrals are the total force, the first and second force moments, respectively. By the same matching process as in Section 4.2, we find the multipole moments,

$$
\begin{gathered}
B_{10}^{(3)}=2 \sqrt{\frac{\pi}{3}} k_{z} \sum_{n=1}^{\infty} \frac{\bar{A}_{0 n}^{(1)}}{j_{0 n}^{2}} \tanh \left(j_{0 n} \ell\right) J_{1}\left(j_{0 n}\right)=k_{z} \bar{B}_{10}^{(3)}, \\
B_{21}^{(5)}=-\mathrm{i} \sqrt{\frac{2 \pi}{15}} k_{x} k_{z} \sum_{n=1}^{\infty} \frac{\bar{A}_{1 n}^{(2)}}{j_{1 n}^{2}} \tanh \left(j_{1 n} \ell\right) J_{2}\left(j_{1 n}\right)=-\mathrm{i} k_{x} k_{z} \bar{B}_{21}^{(5)}, \\
B_{30}^{(5)}=-\frac{1}{5} \sqrt{\frac{\pi}{7}} k_{z} \sum_{n=1}^{\infty} \frac{\bar{A}_{0 n}^{(1)}}{j_{0 n}^{2}} \tanh \left(j_{0 n} \ell\right)\left(J_{1}\left(j_{0 n}\right)-\frac{2 J_{2}\left(j_{0 n}\right)}{j_{0 n}}\right) \\
=-k_{z} \bar{B}_{30}^{(5)},
\end{gathered}
$$

where coefficients $\bar{A}_{0 n}^{(1)}$ and $\bar{A}_{1 n}^{(2)}$ are solved in Eq. (50). The multipole structure of the scattering waves contains the leading dipole $(1,0)$, of $O\left(\varepsilon^{3}\right)$, and is followed by the quadrupole $(2,1)$ and the octupole $(3,0)$ of $O\left(\varepsilon^{5}\right)$. Similar to their rigid boundary counterparts, the moment coefficients $\bar{B}_{10}^{(3)}, \bar{B}_{21}^{(5)}$ and $\bar{B}_{30}^{(5)}$ are functions that depend on the pore depth.

\subsection{Calculation and discussion}

We solve the inner flow for the leading two orders similar to Section 3 and investigate the leading multipole structure of the scattering wave. The coefficient set of each equation systems has the same characteristics as those in Fig. 2(b). We choose $N=120$ for calculations in this section, which produces an absolute error no greater than approximately 0.01 .

The total pressure contour of the first-order-inner flow is sketched in Fig. 9(a). The pressure is normalized by the wavenumber factor $k_{z}$. Subtracting the external incident and reflected wave field, we visualize the inner doublet in Fig. 9(b). It is a dipole aligned vertically to the flanged surface. It has no azimuthal dependence, i.e. $m=0$, and its strength is proportional to the total pressure force exerted on the pore exit plane. The secondorder inner flow, normalized by the factors $-\mathrm{i} k_{x} k_{z}$, is plotted in Fig. 10. The flow is a quadrulet of $(2,1)$. The flow is induced by the non-zero factor $k_{x} k_{z}$, and its strength is related to the $x$-moment of the force at the exit.

The outer field region is the resultant field of the multipole scattering waves. The solution leads by the dipole $(1,0)$ of $O\left(\varepsilon^{3}\right)$. It is followed by the quadrupole $(2,1)$ and the octupole $(3,0)$ of $O\left(\varepsilon^{5}\right)$. They are summarized in 
(a)

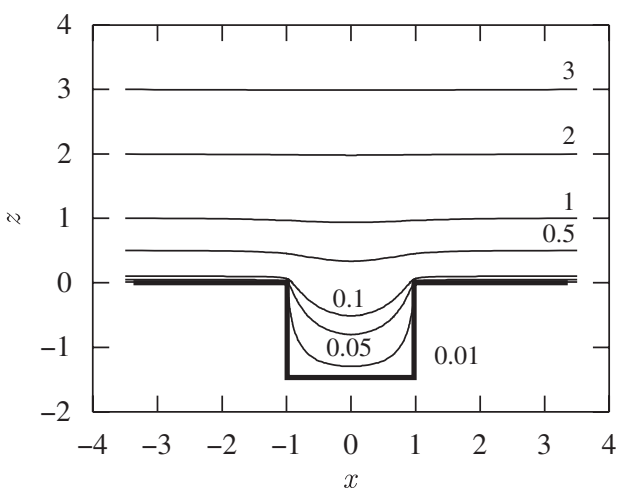

(b)

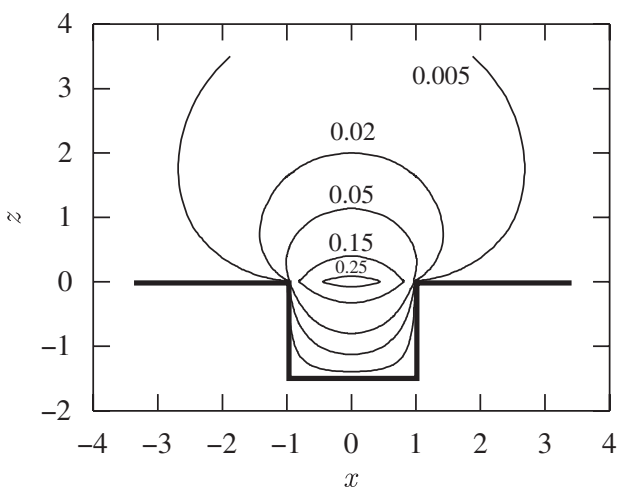

Fig. 9. Pressure contour of (a) total inner pressure, $\mathrm{i} p^{(1)} / k_{z}$ and (b) inner doublet $(1,0), \mathrm{i} p^{(1)} / k_{z}$ and $\mathrm{i} p^{(1)} / k_{z}-z$ for $z<0$ and $z \geqslant 0$.

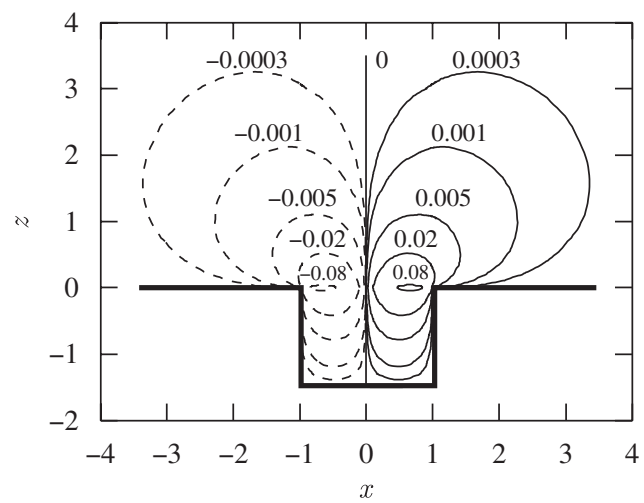

Fig. 10. Pressure contour of inner quadrulet $(2,1), p^{(2)} /\left(k_{x} k_{z}\right)$ and $p^{(2)} /\left(k_{x} k_{z}\right)-x z$ for $z<0$ and $z \geqslant 0$.

Table 2

Multipole structures for the pressure-release surface

\begin{tabular}{lcc}
\hline Multipole & $O\left(\varepsilon^{3}\right)$ & $O\left(\varepsilon^{5}\right)$ \\
\hline Dipole (1,0) & $k_{z} \bar{B}_{10}^{(3)}$ & \\
Quadrupole (2,1) & & $-\mathrm{i} k_{x} k_{z} \bar{B}_{21}^{(5)}$ \\
Octupole (3,0) & $-k_{z} \bar{B}_{30}^{(5)}$ \\
\hline
\end{tabular}

Table 2. The effective moment coefficients are plotted in Figs. 11 and 12, respectively. They all vanish when the pore depth approaches zero and well asymptote to their saturated constants when the depth is larger than about one pore radius.

The effect of the incident angle is extracted as multiplier factors $k_{z}$ and $-\mathrm{i} k_{x} k_{z}$ to the effective moment coefficients. They are both zero when the incident wave is parallel to the surface. This is realizable because the external wave vanishes on the plane surface and, as a result, induces no scattering field at the two solved magnitudes of order. The scattering field in this case degenerates further into higher orders and is not a concern of the present investigation. The quadrupole $(2,1)$ depends on the factor $k_{x} k_{z}$. Therefore, it does not appear when the wave is normally incident on the pore and reaches its maximum when the incident angle is at $45^{\circ}$. 
(a)

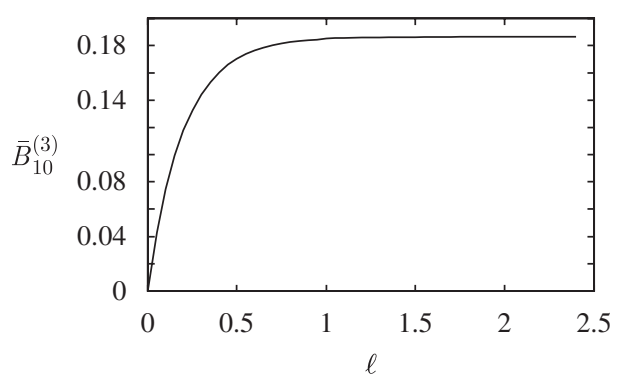

(b)

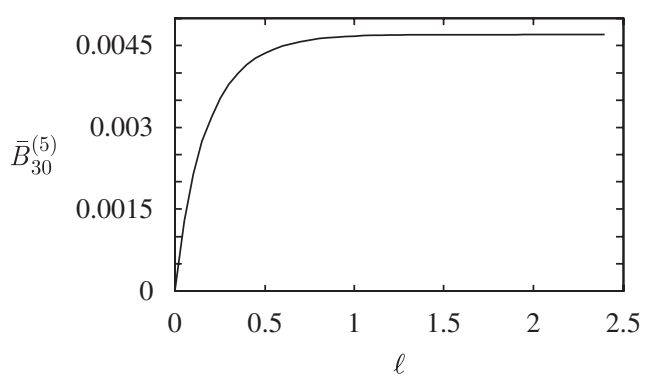

Fig. 11. The effective multipole moment coefficient versus pore depth of (a) dipole (1,0), Eq. (53) and (b) octupole (3,0), Eq. (55).

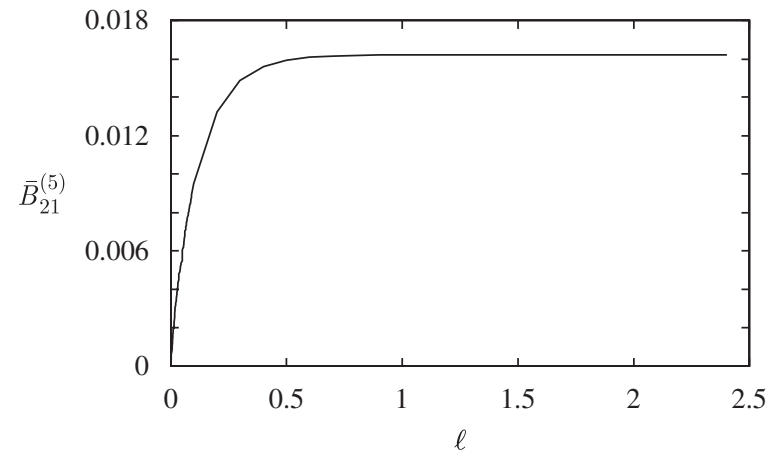

Fig. 12. The effective moment coefficient versus pore depth of quadrupole $(2,1)$, Eq. (54).

\section{Conclusion}

The scattering wave field of acoustic waves incident obliquely on a compact pore in a semi-infinite domain is solved analytically using the method of matched asymptotic expansion. The acoustic wave length is assumed to be much larger than the radius of the pore. The scattering field is, therefore, divided into an inner flow field and an outer wave field. The inner field, described by the Laplace or Poisson equation, is solved by the method developed by Fabrikant, while the outer scattering field is described by the wave equation. By the matching procedures, we obtained uniformly valid multipole expansion solutions for both rigid and pressure-release boundaries. They are summarized in Tables 1 and 2 . The only geometric effect of the pore after normalization with respect to the pore radius is the depth. It modifies the wave scattering through its influence on the effective moment coefficients, $\bar{B}_{m n}^{(3,5)}$. The coefficients are shown to vanish altogether when $\ell=0$ and to asymptote to constant values when $\ell$ exceeds about 1 . The dependence of the incident angle is extracted as multiplier factors of the multipole moment coefficients.

For the rigid condition, there is a monopole which originates from the second-order inner flow due to the small but non-negligible compressibility. Its strength is linearly proportional to the storage capability of the pore, the depth, owing to the extra compressed mass being stored in the pore, as discussed in Section 3.5. The pore volume also raises two accompanying components of the quadrupole $(2,0)$, whose strengths scale linearly with the pore depth. The other multipoles are all induced by the incident angle and reach maxima when the incident wave is parallel to the surface. They include the dipole $(1,0)$, the quadrupoles $(2,0)$ and $(2,2)$ and the octupole $(3,1)$. The multipole strengths are related to the moments of the mass flux entering the pore.

On the other hand, the multipole structure is changed accordingly for the pressure-release condition. The leading field is a dipole perpendicular to the pore exit and is followed by the quadrupole $(2,1)$ and the octupole $(3,0)$. The strength of the dipole is proportional to the total pressure force exerted on the pore exit plane, and those of the quadrupole and the octupole are proportional to the $x$-moment and the second radial moment of 
the force on the exit plane, respectively. These waves all have a common incident angle dependence of $k_{z}$ and disappear from the investigated orders of magnitude when the incident wave is parallel to the flanged surface, Section 4.3. The quadrupole $(2,1)$ scales on $k_{x} k_{z}$ and exhibits a maximum when the incident angle is at $45^{\circ}$. There are no compressibility terms arising in this case, as it did in the rigid boundary one.

Although only a scalar equation is considered in the present study, the current theory can be applied to more sophisticated problems. A broad family of scattering problems are currently under investigation, notable examples being electromagnetic wave scattering and the anisotropic effect of elliptical pores. In the future, the expansion enables us to treat the pore as an individual scatterer and, thus, to investigate the wave fields from surfaces with various pore structures by formulating the mutual interactions as multiple scattering processes: see Ishimaru [14].

\section{Appendix A. Fundamentals of potential theory of mixed boundary value problems}

We provide brief explanations of the two major prerequisites of Fabrikant's potential theory of mixed boundary value problems. They are the Poisson operator $\mathscr{L}(\lambda)$ and the integral representations of the inverse of a spatial distance. Derivation of the double integral of Eq. (7) is also shown in this section as a concluding application demonstration of the two ingredients.

Consider a function $f(r, \phi)$ with a periodic $2 \pi$. This function can be associated with Fourier coefficient $f_{m}(r)$, where

$$
f_{m}(r)=\frac{1}{2 \pi} \int_{0}^{2 \pi} \mathrm{e}^{\mathrm{i} m \phi} f(r, \phi) \mathrm{d} \phi .
$$

Define the Poisson operator $\mathscr{L}(\lambda)$ for a complex $\lambda$ such that

$$
\mathscr{L}(\lambda) f_{m}(r)=\lambda^{|m|} f_{m}(r)
$$

Consequently, we have the operator properties $\mathscr{L}\left(\lambda_{1}\right) \mathscr{L}\left(\lambda_{2}\right)=\mathscr{L}\left(\lambda_{1} \lambda_{2}\right)$ and $\mathscr{L}(1)=1$.

Now, look at

$$
\begin{aligned}
\mathscr{L}(\lambda) f(r, \phi) & =\sum_{m=-\infty}^{\infty} \lambda^{|m|} f_{m}(r) \mathrm{e}^{-\mathrm{i} m \phi} \\
& =\frac{1}{2 \pi} \int_{0}^{2 \pi} \mathrm{d} \phi_{0} f\left(r, \phi_{0}\right) \sum_{m=-\infty}^{\infty} \lambda^{|m|} \mathrm{e}^{\mathrm{i} m\left(\phi-\phi_{0}\right)} .
\end{aligned}
$$

The series in the integral can be evaluated analytically for $|\lambda| \leqslant 1$ to

$$
\Lambda\left(\lambda, \phi_{0}-\phi\right)=\frac{\left(1-\lambda^{2}\right)}{1+\lambda^{2}-2 \lambda \cos \left(\phi_{0}-\phi\right)}
$$

and the whole equation is recast into

$$
\mathscr{L}(\lambda) f(r, \phi)=\frac{1}{2 \pi} \int_{0}^{2 \pi} \Lambda\left(\lambda, \phi-\phi_{0}\right) f\left(r, \phi_{0}\right) \mathrm{d} \phi_{0} .
$$

This equation defines the analytical form of the operator $\mathscr{L}(\lambda)$ for $|\lambda| \leqslant 1$.

On the other hand, the square of the distance between two points $(\rho, \phi, z)$ and $\left(\rho_{0}, \phi_{0}, 0\right)$ using the polar coordinate defined in Fig. 1. is

$$
\mathrm{s}^{2}=\rho^{2}+\rho_{0}^{2}-2 \rho \rho_{0} \cos \left(\phi-\phi_{0}\right)+z^{2} .
$$

We define the new variables $l_{1}$ and $l_{2}$ such that the distance can be represented as

$$
\mathrm{s}^{2}=l_{1}^{2}+l_{2}^{2}-2 l_{1} l_{2} \cos \left(\phi-\phi_{0}\right) \text {. }
$$

This leads to the two equations

$$
l_{1}^{2}+l_{2}^{2}=\rho^{2}+\rho_{0}^{2}+z^{2} \quad \text { and } \quad l_{1} l_{2}=\rho \rho_{0}
$$


with the solution

$$
\begin{aligned}
& l_{1}=l_{1}\left(\rho_{0}, \rho, z\right)=\frac{1}{2}\left(\sqrt{\left(\rho+\rho_{0}\right)^{2}+z^{2}}-\sqrt{\left(\rho-\rho_{0}\right)^{2}+z^{2}}\right), \\
& l_{2}=l_{2}\left(\rho_{0}, \rho, z\right)=\frac{1}{2}\left(\sqrt{\left(\rho+\rho_{0}\right)^{2}+z^{2}}+\sqrt{\left(\rho-\rho_{0}\right)^{2}+z^{2}}\right) .
\end{aligned}
$$

Variable $l_{1}$ is the arithmetic average of the difference between the longest and the shortest possible distances of the two points and $l_{2}$ is the average of the sum of the two extreme distances when $\rho_{0}$ is kept constant and $\phi_{0}$ varies within $[0,2 \pi]$.

Starting with the identity, e.g. formula (3.249) of Ref. [12]

$$
\frac{1}{\mathrm{~s}}=\frac{2}{\pi} \int_{0}^{\infty} \frac{\mathrm{d} \eta}{\mathrm{s}^{2}+\eta^{2}}
$$

and making the change of variable

$$
\eta=\sqrt{l_{1}^{2}-x^{2}} \sqrt{l_{2}^{2}-x^{2}} / x
$$

we have

$$
\Lambda\left(\frac{x^{2}}{\rho \rho_{0}}, \phi-\phi_{0}\right)=-\frac{x \eta}{\mathrm{s}^{2}+\eta^{2}} \frac{\mathrm{d} \eta}{\mathrm{d} x},
$$

where $\Lambda\left(\lambda, \phi-\phi_{0}\right)$ is defined in Eq. (A.2). Substituting Eqs. (A.5) and (A.6) into the identity (A.4), we obtain the first integral representation of the inverse of the distance

$$
\frac{1}{\mathrm{~s}}=\frac{2}{\pi} \int_{0}^{l_{1}} \frac{\Lambda\left(x^{2} / \rho \rho_{0}, \phi-\phi_{0}\right) \mathrm{d} x}{\sqrt{l_{1}^{2}-x^{2}} \sqrt{l_{2}^{2}-x^{2}}},
$$

where we notice that $x$ in the above expression is the dummy integration variable.

Alternatively, if we redefine $\eta$ to be

$$
\eta=\sqrt{x^{2}-l_{1}^{2}} \sqrt{x^{2}-l_{2}^{2}} / x
$$

and follow similar procedures for the change of variable, we acquire the second integral representation of $1 / \mathrm{s}$ as

$$
\frac{1}{\mathrm{~s}}=\frac{2}{\pi} \int_{l_{2}}^{\infty} \frac{\Lambda\left(\rho \rho_{0} / x^{2}, \phi-\phi_{0}\right) \mathrm{d} x}{\sqrt{x^{2}-l_{1}^{2}} \sqrt{x^{2}-l_{2}^{2}}} .
$$

The benefit of adopting these representations, or decompositions, Eqs. (A.7) and (A.8), is that the interwoven variable $\phi-\phi_{0}$ is absorbed in function $\Lambda$. This makes many multiple integrations analytically possible. These decompositions also have other good mathematical properties which are discussed in Ref. [10].

We often need to bring a field point to the $z=0$ plane in formulating our integral equations. In the limiting case, the variables $l_{1}$ and $l_{2}$ reduce to

$$
\begin{aligned}
& \bar{l}_{1}=\lim _{z \rightarrow 0} l_{1}\left(\rho_{0}, \rho, z\right)=\min \left(\rho_{0}, \rho\right), \\
& \bar{l}_{2}=\lim _{z \rightarrow 0} l_{2}\left(\rho_{0}, \rho, z\right)=\max \left(\rho_{0}, \rho\right) .
\end{aligned}
$$

Having these prerequisites, the derivation of the scattering term of Eq. (6) to that of Eq. (7) is straightforward. Using the first representation (A.7) and combining the $\phi_{0}$-integral with function $\Lambda$, we have

$$
\left.p_{\mathrm{sc}}^{(1)}\right|^{<}=-\left.\frac{2}{\pi} \int_{0}^{1} \mathrm{~d} \rho_{0} \rho_{0} \int_{0}^{\bar{l}_{1}} \frac{1}{\sqrt{\bar{l}_{1}^{2}-x^{2}} \sqrt{\bar{l}_{2}^{2}-x^{2}}} \mathscr{L}\left(\frac{x^{2}}{\rho \rho_{0}}\right) \frac{\partial p}{\partial z}\right|^{<} \mathrm{d} x .
$$


(a)

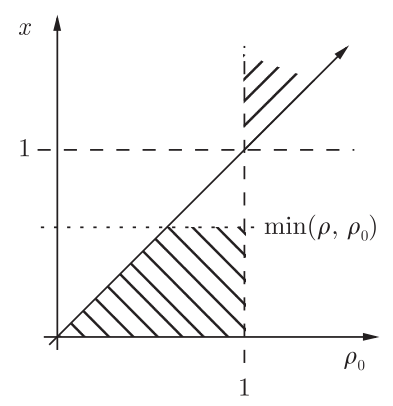

(b)

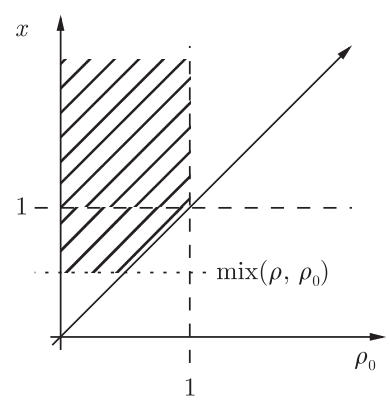

Fig. A.1. Integration domain.

The integration domain is the lower shaded area in Fig. A.1(a). Changing the order of integration, we obtain Eq. (7).

\section{Appendix B. Miscellaneous details}

\section{B.1. Derivation of Eq. (10)}

First of all, we recall the right-hand side of Eq. (6), after substituting Eq. (7) for the scattering wave, and applying the first inverse operator (8)

$$
\begin{aligned}
& \mathscr{L}\left(\frac{1}{r}\right) \frac{\mathrm{d}}{\mathrm{d} r} \int_{0}^{r} \frac{\rho \mathrm{d} \rho}{\sqrt{r^{2}-\rho^{2}}} \mathscr{L}(\rho) \\
& \quad \times\left\{\mathrm{i} k_{x} \rho \cos \phi-\left.\frac{2}{\pi} \int_{0}^{\rho} \frac{\mathrm{d} x}{\sqrt{\rho^{2}-x^{2}}} \int_{x}^{1} \frac{\rho_{0} \mathrm{~d} \rho_{0}}{\sqrt{\rho_{0}^{2}-x^{2}}} \mathscr{L}\left(\frac{x^{2}}{\rho \rho_{0}}\right) \frac{\partial p}{\partial z_{0}}\right|^{<}\right\} .
\end{aligned}
$$

With Eq. (A.1), we have $\mathscr{L}(\rho) \cos \phi=\rho \cos \phi$. Therefore, the first term of Eq. (B.1) becomes

$$
\mathrm{i} k_{x} \cos \phi \frac{\mathrm{d}}{r \mathrm{~d} r} \int_{0}^{r} \frac{\rho^{3} \mathrm{~d} \rho}{\sqrt{r^{2}-\rho^{2}}},
$$

which can be evaluated by parts

$$
\mathrm{i} k_{x} \cos \phi \frac{\mathrm{d}}{r \mathrm{~d} r}\left(-\left.\rho^{2} \sqrt{r^{2}-\rho^{2}}\right|_{\rho=0} ^{r}+2 \int_{0}^{r} \rho \sqrt{r^{2}-\rho^{2}} \mathrm{~d} \rho\right) .
$$

Carrying out the integral, we have the field, $2 \mathrm{i} k_{x} r \cos \phi$, after the first inversion. Application of the second inverse operator, Eq. (9), is similar, and after a few elementary integration steps, we have the inversed external field,

$$
-2 \mathrm{i} k_{x} t^{2}\left(1-t^{2}\right)^{-1 / 2} \cos \phi
$$

The same procedures are applied on other external fields with azimuthal modes $m=0$ and 2 , such as those in Eqs. (19) and (49).

The second term of Eq. (B.1) reads

$$
-\frac{2}{\pi} \mathscr{L}\left(\frac{1}{r}\right) \frac{\mathrm{d}}{\mathrm{d} r} \int_{0}^{r} \frac{\rho \mathrm{d} \rho}{\sqrt{r^{2}-\rho^{2}}} \int_{0}^{\rho} \frac{\mathrm{d} x}{\sqrt{\rho^{2}-x^{2}}} \underbrace{\left.\int_{x}^{1} \frac{\rho_{0} \mathrm{~d} \rho_{0}}{\sqrt{\rho_{0}^{2}-x^{2}}} \mathscr{L}\left(\frac{x^{2}}{\rho_{0}}\right) \frac{\partial p\left(\rho_{0}, \phi, z_{0}\right)}{\partial z_{0}}\right|^{<}}_{\mathscr{F}(x, \phi)} .
$$


Provided that $\mathscr{F}(x, \phi)$ belongs to $L^{1}$, which holds for physics, the first two integrals can change the order of operation,

$$
-\frac{2}{\pi} \mathscr{L}\left(\frac{1}{r}\right) \frac{\mathrm{d}}{\mathrm{d} r} \int_{0} \mathrm{~d} x \mathscr{F}(x, \phi) \int_{x}^{r} \frac{\rho \mathrm{d} \rho}{\sqrt{r^{2}-\rho^{2}} \sqrt{\rho^{2}-x^{2}}}
$$

with the inner integral trivial. Resuming the definition of $\mathscr{F}(x, \phi)$ and using the l'Hopital rule, we have

$$
-\left.\int_{r}^{1} \frac{\rho_{0} \mathrm{~d} \rho_{0}}{\sqrt{\rho_{0}^{2}-r^{2}}} \mathscr{L}\left(\frac{r}{\rho_{0}}\right) \frac{\partial p}{\partial z_{0}}\right|^{<}
$$

Next, applying the second inverse operator on Eq. (B.3),

$$
-\left.\mathscr{L}(t) \frac{\mathrm{d}}{\mathrm{d} t} \int_{t}^{1} \frac{r \mathrm{~d} r}{\sqrt{r^{2}-t^{2}}} \int_{r}^{1} \frac{\rho_{0} \mathrm{~d} \rho_{0}}{\sqrt{\rho_{0}^{2}-r^{2}}} \mathscr{L}\left(\frac{1}{\rho_{0}}\right) \frac{\partial p}{\partial z_{0}}\right|^{<}
$$

and changing the order of integration, we have the resultant integrals integrable which yield

$$
\left.\frac{\pi}{2} t \frac{\partial p\left(t, \phi, z_{0}\right)}{\partial z_{0}}\right|^{<} \text {. }
$$

Expressions (B.2) and (B.4) are the inversed terms in Eq. (10).

\section{B.2. Inversion of Eq. (42)}

As described in Appendix A, there are two alternative ways to represent the integrals of Eq. (42). The most convenient choice is to make the final form of the outermost integrals on both sides of the equation over the same interval, such that the inversion can be done simultaneously. For this purpose, we recast Eq. (42) into

$$
\begin{aligned}
& \left.\int_{1}^{\infty} \mathrm{d} \rho_{0} \rho_{0} \int_{\bar{l}_{2}}^{\infty} \frac{\mathrm{d} x}{\sqrt{x^{2}-\bar{l}_{1}^{2}} \sqrt{x^{2}-\bar{l}_{2}^{2}}} \mathscr{L}\left(\frac{\rho \rho_{0}}{x^{2}}\right) \frac{\partial p_{\mathrm{sc}}^{(1,2)}}{\partial z_{0}}\right|^{>} \\
& =-\left.\int_{0}^{1} \mathrm{~d} \rho_{0} \rho_{0} \int_{\bar{l}_{2}}^{\infty} \frac{\mathrm{d} x}{\sqrt{x^{2}-\bar{l}_{1}^{2}} \sqrt{x^{2}-\bar{l}_{2}^{2}}} \mathscr{L}\left(\frac{\rho \rho_{0}}{x^{2}}\right) \frac{\partial p_{\mathrm{sc}}^{(1,2)}}{\partial z_{0}}\right|^{<},
\end{aligned}
$$

where $\bar{l}_{1}$ and $\bar{l}_{2}$ are given in Eq. (A.9). The integral domains for the left- and right-hand sides are, respectively, the lower and upper shaded regions in Figs. A.1(a). Changingg the order of the integration, we obtain Eq. (43) straightforwardly.

Since both sides of the above equation are in the same form, we only demonstrate the application of the first inverse operator, Eq. $(44)_{1}$, on the left-hand side term. It reads

$$
\mathscr{L}(t) \frac{\mathrm{d}}{\mathrm{d} t} \int_{t}^{\infty} \frac{\rho \mathrm{d} \rho}{\sqrt{\rho^{2}-t^{2}}} \int_{\rho}^{\infty} \frac{\mathrm{d} x}{\sqrt{x^{2}-\rho^{2}}} \underbrace{\left.\int_{1}^{x} \frac{\rho_{0} \mathrm{~d} \rho_{0}}{\sqrt{x^{2}-\rho_{0}^{2}}} \mathscr{L}\left(\frac{\rho_{0}}{x^{2}}\right) \frac{\partial p_{\mathrm{sc}}^{(1,2)}}{\partial z}\right|^{>}}_{\mathscr{F}(x, \phi)}
$$

and following

$$
\mathscr{L}(t) \frac{\mathrm{d}}{\mathrm{d} t} \int_{t}^{\infty} \mathrm{d} x \mathscr{F}(x, \phi) \int_{t}^{x} \frac{\rho \mathrm{d} \rho}{\sqrt{\rho^{2}-t^{2}} \sqrt{x^{2}-\rho^{2}}} .
$$

The inner integration is again integrable and the full equation after the first inversion becomes

$$
\left.\mathscr{L}\left(\frac{\rho_{0}}{t}\right) \int_{1}^{t} \frac{\rho_{0} \mathrm{~d} \rho_{0}}{\sqrt{t^{2}-\rho_{0}^{2}}} \frac{\partial p_{\mathrm{sc}}^{(1,2)}}{\partial z_{0}}\right|^{>}=-\left.\mathscr{L}\left(\frac{\rho_{0}}{t}\right) \int_{0}^{1} \frac{\rho_{0} \mathrm{~d} \rho_{0}}{\sqrt{t^{2}-\rho_{0}^{2}}} \frac{\partial p_{\mathrm{sc}}^{(1,2)}}{\partial z_{0}}\right|^{<} .
$$


Next, applying the second inverse operator $(44)_{2}$ and changing the order of integration, we have

$$
\begin{gathered}
\left.\mathscr{L}\left(\frac{1}{\rho}\right) \frac{\mathrm{d}}{\mathrm{d} \rho} \int_{1}^{\rho} \mathrm{d} \rho_{0} \rho_{0} \mathscr{L}\left(\rho_{0}\right) \frac{\partial p_{\mathrm{sc}}^{(1,2)}}{\partial z_{0}}\right|^{>} \int_{\rho_{0}}^{\rho} \frac{t \mathrm{~d} t}{\sqrt{\rho^{2}-t^{2}} \sqrt{t^{2}-\rho_{0}^{2}}} \\
=-\left.\mathscr{L}\left(\frac{1}{\rho}\right) \int_{0}^{1} \mathrm{~d} \rho_{0} \rho_{0} \mathscr{L}\left(\rho_{0}\right) \frac{\partial p_{\mathrm{sc}}^{(1,2)}}{\partial z_{0}}\right|^{<} \frac{\mathrm{d}}{\mathrm{d} \rho} \int_{1}^{\rho} \frac{t \mathrm{~d} t}{\sqrt{\rho^{2}-t^{2}} \sqrt{t^{2}-\rho_{0}^{2}}} .
\end{gathered}
$$

The inner integrals are elementary and, therefore, we reach Eq. (45).

\section{B.3. Derivation of Eq. (46)}

We start from the total pressure at the pore exit. The last term of Eq. (41) on the right-hand side with Eq. (A.8) and the definition (A.2) can be recast into

$$
-\left.\frac{2}{\pi} \int_{1}^{\infty} \frac{\mathrm{d} x}{\sqrt{x^{2}-\rho^{2}}} \int_{1}^{x} \frac{\rho_{0} \mathrm{~d} \rho_{0}}{\sqrt{x^{2}-\rho_{0}^{2}}} \mathscr{L}\left(\frac{\rho \rho_{0}}{x^{2}}\right) \frac{\partial p_{\mathrm{sc}}^{(1,2)}}{\partial z_{0}}\right|^{>} .
$$

Substituting the solved pressure derivative on the flanged surface, Eq. (45), this term becomes

$$
\left.\frac{4}{\pi^{2}} \int_{1}^{\infty} \frac{\mathrm{d} x}{\sqrt{x^{2}-\rho^{2}}} \int_{1}^{x} \frac{\rho_{0} \mathrm{~d} \rho_{0}}{\sqrt{x^{2}-\rho_{0}^{2}} \sqrt{\rho_{0}^{2}-1}} \int_{0}^{1} \frac{y \sqrt{1-y^{2}} \mathrm{~d} y}{\rho_{0}^{2}-y^{2}} \mathscr{L}\left(\frac{\rho y}{x^{2}}\right) \frac{\partial p_{\mathrm{sc}}^{(1,2)}}{\partial z_{0}}\right|^{<} .
$$

The inner two integrals are inter-changeable, and so

$$
\left.\frac{4}{\pi^{2}} \int_{1}^{\infty} \frac{\mathrm{d} x}{\sqrt{x^{2}-\rho^{2}}} \int_{0}^{1} \mathrm{~d} y y \sqrt{1-y^{2}} \mathscr{L}\left(\frac{\rho y}{x^{2}}\right) \frac{\partial p_{\mathrm{sc}}^{(1,2)}}{\partial z_{0}}\right|^{<} \int_{1}^{x} \frac{\rho_{0} \mathrm{~d} \rho_{0}}{\sqrt{x^{2}-\rho_{0}^{2}} \sqrt{\rho_{0}^{2}-1}\left(\rho_{0}^{2}-y^{2}\right)} .
$$

Carrying out the innermost integration, we have

$$
\left.\frac{2}{\pi} \int_{1}^{\infty} \frac{\mathrm{d} x}{\sqrt{x^{2}-\rho^{2}}} \int_{0}^{1} \frac{y \mathrm{~d} y}{\sqrt{x^{2}-y^{2}}} \mathscr{L}\left(\frac{\rho y}{x^{2}}\right) \frac{\partial p_{\mathrm{sc}}^{(1,2)}}{\partial z_{0}}\right|^{<} .
$$

On the other hand, the first term on the right-hand side of Eq. (41) can be recast into

$$
-\left.\frac{2}{\pi} \int_{0}^{1} \mathrm{~d} \rho_{0} \rho_{0} \int_{\bar{l}_{2}}^{\infty} \frac{\mathrm{d} x}{\sqrt{x^{2}-\rho^{2}} \sqrt{x^{2}-\rho_{0}^{2}}} \mathscr{L}\left(\frac{\rho \rho_{0}}{x^{2}}\right) \frac{\partial p_{\mathrm{sc}}^{(1,2)}}{\partial z_{0}}\right|^{<}
$$

with the integration domain in Fig. A.1(b). It follows straightforwardly the change of integration order

$$
-\left.\frac{2}{\pi} \int_{\rho}^{\infty} \frac{\mathrm{d} x}{\sqrt{x^{2}-\rho^{2}}} \int_{0}^{\min (x, 1)} \frac{\rho_{0} \mathrm{~d} \rho_{0}}{\sqrt{x^{2}-\rho_{0}^{2}}} \mathscr{L}\left(\frac{\rho \rho_{0}}{x^{2}}\right) \frac{\partial p_{\mathrm{sc}}^{(1,2)}}{\partial z_{0}}\right|^{<},
$$

which is ready to be combined with Eq. (B.5). Finally, we have Eq. (46) after substituting the continuity conditions of the pressure at the pore exit.

\section{References}

[1] R. Sato, H. Shirai, Acoustic analysis of plane-wave transmission through a duct of a thick wall, Journal of the Acoustical Society of America 100 (4) (1996) 2596.

[2] E.L. Shenderov, Scattering of a plane sound wave at a semi-infinite waveguide with a stiff flange, Acoustical Physics 44 (1) (1998) 88-96.

[3] R.W. Scharstein, A.M.J. Davis, Matched asymptotic expansion for the low-frequency scattering by a semi-circular trough in a ground plane, IEEE Transactions on Antennas and Propagation 48 (2000) 801-811. 
[4] F.J. Garcia-Vidal, E. Moreno, J.A. Porto, L. Martin-Moreno, Transmission of light through a single rectangular hole, Physical Review Letters 95 (2005) 103901.

[5] F.J. Garcia de Abajo, J.J. Saenz, Electromagnetic surface modes in structured perfect-conductor surfaces, Physical Review Letters 95 (4) (2005) 233901.

[6] A.D. Pierce, Acoustics: an Introduction to its Physical Principles and Applications, McGraw-Hill, New York, 1981.

[7] D.G. Crighton, A.P. Dowling, J.E. Ffowcs Williams, M. Heckl, F.G. Leppington, Modern Methods in Analytical Acoustics, Springer, Berlin, 1992, pp. 168-208 (Chapter 13).

[8] C.Y. Kuo, A.P. Dowling, Acoustics of a two-dimensional compact jet impinging normally onto a flat plate, Journal of Fluid Mechanics 414 (2000) 251-284.

[9] I.N. Sneddon, Mixed Boundary Value Problems in Potential Theory, Wiley, New York, 1966.

[10] V.I. Fabrikant, Applications of Potential Theory in Mechanics, a Selection of New Results, Kluwer, Dordrecht, 1989.

[11] R. Bracewell, The Fourier Transform and Its Applications, third ed., McGraw-Hill, New York, 1999.

[12] I.S. Gradshteyn, I.M. Ryzhik, Table of Integrals, Series, and Products, Academic, 1994.

[13] M. Van Dyke, Perturbation Methods in Fluid Mechanics, Parabolic, Stanford, 1975.

[14] A. Ishimaru, Wave Propagation and Scattering in Random Media, IEEE, New York, 1994. 